\title{
Leading order multi-soft behaviors of tree amplitudes in NLSM
}

\author{
Yi-Jian $\mathbf{D u}^{a}$ and Hui $\mathbf{L u o}^{b}$ \\ ${ }^{a}$ Center for Theoretical Physics, School of Physics and Technology, Wuhan University, \\ 299 Bayi Road, Wuhan 430072, China \\ ${ }^{b}$ II. Institut für Theoretische Physik, Universität Hamburg, \\ Luruper Chaussee 149, D-22761 Hamburg, Germany \\ E-mail: yijian.du@whu.edu.cn, hui.luo@desy.de
}

ABSTRACT: In this paper, we investigate multi-soft behaviors of tree amplitudes in nonlinear sigma model (NLSM). The leading behaviors of amplitudes with odd number of all-adjacent soft pions are zero. We further propose and prove that leading soft factors of amplitudes with even number all-adjacent soft pions can be expressed in terms of products of the leading order Berends-Giele sub-currents in Cayley parametrization. Each subcurrent in the expression contains at most one hard pion. Discussions are generalized to amplitudes containing arbitrary number of nonadjacent soft blocks: the leading behaviors of amplitudes where at least one soft block has odd number of adjacent soft pions are zero; the leading soft factors for amplitudes where all soft blocks containing even number of soft pions are given by products of soft factors for these blocks.

KeYwords: Global Symmetries, Scattering Amplitudes

ARXIV EPRINT: 1611.07479 


\section{Contents}

1 Introduction 1

2 A review of off-shell and on-shell recursions in NLSM 4

2.1 Feynman rules and Berends-Giele recursion relations 4

2.2 On-shell recursion relations of effective theories 5

3 Amplitudes with adjacent soft pions of odd number 5

$\begin{array}{lll}3.1 & \text { On-shell recursion approach } & 6\end{array}$

$\begin{array}{lll}3.2 & \text { Berends-Giele recursion approach } & 7\end{array}$

4 Amplitudes with adjacent soft pions of even number 10

$\begin{array}{ll}\text { 4.1 One soft pion is adjacent to the off-shell line } & 10\end{array}$

$\begin{array}{lll}4.2 & \text { All soft pions are nonadjacent to the off-shell line } & 13\end{array}$

4.3 The behavior of on-shell amplitudes with even number of adjacent soft pions 14

$\begin{array}{ll}4.4 \text { Quadruple soft limits } & 15\end{array}$

5 Amplitudes with nonadjacent soft blocks $\quad 16$

6 Conclusion 16

$\begin{array}{ll}\text { A The proof of eq. (4.9) } & 17\end{array}$

$\begin{array}{ll}\text { A.1 An example } & 17\end{array}$

A.2 General proof 20

$\begin{array}{ll}\text { B Sextuple behavior } & 24\end{array}$

\section{Introduction}

Symmetry is one of the most critical topic in physics. Refer to the global symmetry, which is alway broken to describe the real world, it can be broken spontaneously by gaining certain vacuum expectation values or explicitly by terms violating the symmetry. In particular, once a global symmetry $G$ of a theory is spontaneously broken into its subgroup $H$, massless Goldstone bosons are in one-to-one correspondence to the broken generators. The unbroken symmetry generators $T_{i}$ and the broken generators $X_{a}$ satisfy the following schematic commutation relations

$$
[T, T] \sim T,[X, T] \sim X,[X, X] \sim T .
$$

This global symmetry $G$ can be realized by properly defined fields of Goldstone bosons, whose behaviors are able to be described by the nonlinear sigma model (NLSM) [1, 2]. Because the scattering amplitudes at any point of the vacuum moduli space should be identical, the vacuum structure after global symmetry spontaneously breaking can be probed by certain structures of scattering amplitudes [3]. 
To reveal states of Hilbert space in different vacua through scattering amplitudes, one should study the behaviors of amplitudes with additional Goldstone bosons of zero momentum by regulating these constant scalar fields with tiny momenta and sending them to zero eventually with a very careful analysis. More precisely speaking, if a theory after symmetry breaking has a continuous vacuum, one vacuum can be arrived by expanding around another one

$$
\left|\Omega_{\theta}\right\rangle=|\Omega\rangle+\left|\Omega^{(1)}\right\rangle+\left|\Omega^{(2)}\right\rangle+\ldots,
$$

where the variation $\left|\Omega^{(n)}\right\rangle$ should contain $n$ soft pions ${ }^{1}$ information. This implies that the vacuum variations might be discovered by studying soft limits of amplitudes. For example, the first order variation can be understood by taking a single soft pion, which vanishes eventually and is well known as "Adler zero" $[4,5]$. The second order variation can be described by double soft pions emissions, which is related to an $\left[X^{\alpha}, X^{\beta}\right]$ transformation on the amplitude of hard pions. Moreover, the double soft limits are studied up to the next leading order recently $[3,6-9] .{ }^{2}$ Along this line, if studying higher order variations, $\left|\Omega^{(3)}\right\rangle$, $\left|\Omega^{(4)}\right\rangle$ and so on, we need to understand multi-soft behaviors of amplitudes.

In the present work, we focus on the non-linear sigma model (NLSM) from spontaneously global symmetry breaking $\mathrm{SU}(N) \times \mathrm{SU}(N) \rightarrow \mathrm{SU}(N)$. Analogous to the color decomposition in Yang-Mills theory, the flavor structure in NLSM can be separated from kinematic factors which are involved in flavor-ordered partial amplitudes. Once we know all multi-soft behaviors for partial amplitudes, we know the behaviors for full amplitudes. To study the multi-soft behaviors of partial amplitudes, we could either take soft limits consecutively or simultaneously. Although there might be symmetry insights for both consecutive and simultaneous soft limits (see e.g, $[16,17]$ ), one can obtain the consecutive soft behaviors by applying simultaneous ones in principle. In this paper, we systematically study all leading order simultaneous soft behaviors of tree-level partial amplitudes $A(1,2, \cdots, 2 n)$ in NLSM with arbitrary number of soft pions. Through denoting the momenta of all soft pions $i \in\{S\}$ by $k_{i}=\tau q_{i}$, the partial amplitude becomes a function of $\tau$, i.e., $A(\tau)$. Thus the soft behavior is described by $A(\tau)$ with $\tau \rightarrow 0$. The possible leading order of $A(\tau)$ in NLSM is the coefficient of $\tau^{0}$ order in Taylor's expansion.

This work is devoted to the study of all the $\tau^{0}$ behaviors of partial amplitudes in NLSM. We first study the behaviors of amplitudes with all-adjacent soft pions. Such amplitudes are classified according to whether the number of soft pions is odd or even. The behaviors are given as follows:

a) The $\tau^{0}$ order of amplitude $A(1 \cdots, a, \widetilde{a+1}, \cdots, a \widetilde{+2 i}+1, a+2 i+2, \cdots, 2 n)$, which contains odd number of all-adjacent soft pions $\widetilde{a+1}, \cdots, a \widetilde{+2 i+1}$, has to vanish. This result will be illustrated via on-shell recursion $[18,19]$ as well as off-shell recursion [20] in NLSM (see [21-24]). When $i=0$, where only one soft pion is contained, the vanishing behavior agrees with Alder's zero [4].

\footnotetext{
${ }^{1}$ Pion is a physical synonym for Goldstone boson. We don't distinguish them in this paper.

${ }^{2}$ Discussions on soft limits in theories with conformal symmetry breaking and supersymmetry breaking can be found in [10-15].
} 
b) The $\tau^{0}$ order of amplitude $A(1, \cdots, a, \widetilde{a+1}, \cdots, \widetilde{a+2 i}, a+2 i+1, \cdots, 2 n)$, which contains even number of all-adjacent soft pions $\widetilde{a+1}, \cdots, \widetilde{a+2 i}$ and at least four hard pions, ${ }^{3}$ is given by

$$
\begin{aligned}
A & (1, \cdots, a, \widetilde{a+1}, \cdots, \widetilde{a+2} i, a+2 i+1, \cdots, 2 n) \\
& =\mathbb{S}_{a, a+2 i+1}^{(0)} A(1, \cdots, a, a+2 i+1, \cdots, 2 n)+\mathcal{O}(\tau),
\end{aligned}
$$

where the soft factor $\mathbb{S}_{a, a+2 i+1}^{(0)}$ is defined as

$$
\mathbb{S}_{a, a+2 i+1}^{(0)}=\sum_{D \in \operatorname{divisions}\{a, \widehat{a+1}, \cdots, a+2 i, a+2 i+1\}}\left(-\frac{1}{2 F^{2}}\right)^{\frac{N(D)-2}{2}} \prod_{j=1}^{N(D)} J^{(0)}\left(D_{j}\right) .
$$

Here, all possible divisions $D$ of the ordered set $\{a \widetilde{a+1}, \cdots, \widetilde{a+2} i, a+2 i+1\}$ such that each subset contains odd number of elements are summed over. Coupling constant is $-\frac{1}{2 F^{2}}$ and $N(D)$ is the number of subset $\left\{D_{j}\right\}$ for a given division $D$. The number of subsets should be more than one. For example, while setting $a=1$ and considering the behavior of amplitudes with four soft pions, we have the following divisions of $\{1, \widetilde{2}, \widetilde{3}, \widetilde{4}, \widetilde{5}, 6\}$

$$
\begin{array}{lll}
\{\{1, \widetilde{2}, \widetilde{3}, \widetilde{4}, \widetilde{5}\},\{6\}\}, & \{\{1, \widetilde{2}, \widetilde{3}\},\{\widetilde{4}, \widetilde{5}, 6\}\}, & \{\{1\},\{\widetilde{2}, \widetilde{3}, \widetilde{4}, \widetilde{5}, 6\}\}, \\
\{\{1, \widetilde{2}, \widetilde{3}\},\{\widetilde{4}\},\{\widetilde{5}\},\{6\}\}, & \{\{1\},\{\widetilde{2}, \widetilde{3}, \widetilde{4}\},\{\widetilde{5}\},\{6\}\}\}, & \{\{1\},\{\widetilde{2}\},\{\widetilde{3}, \widetilde{4}, \widetilde{5}\},\{6\}\}, \\
\{\{1\},\{\widetilde{2}\},\{\widetilde{3}\},\{\widetilde{4}, \widetilde{5}, 6\}\}, & \{\{1\},\{\widetilde{2}\},\{\widetilde{3}\},\{\widetilde{4}\},\{\widetilde{5}\},\{6\}\} . &
\end{array}
$$

In eq. $(1.4), J^{(0)}\left(D_{j}\right)$ denotes the $\tau^{0}$ order of Berends-Giele currents $J\left(D_{j}\right)$ of $\mathrm{U}(N)$ NLSM (The on-shell amplitudes of $\mathrm{U}(N)$ and $\mathrm{SU}(N)$ NLSM are equivalent) in Cayley parametrization [22]. From the definition of divisions, we know that the even number all-adjacent soft behavior is expressed by the leading orders of two kinds of Berends-Giele sub-currents: i) sub-currents containing only soft external pions, e.g., $J^{(0)}(\widetilde{2}, \widetilde{3}, \widetilde{4})$ from the division $\{\{1\},\{\widetilde{2}, \widetilde{3}, \widetilde{4}\},\{\widetilde{5}\},\{6\}\}\}$, ii) sub-currents where only the first or last pion is hard and all others are soft, e.g., $J^{(0)}(1, \widetilde{2}, \widetilde{3})$ from the division $\{\{1, \widetilde{2}, \widetilde{3}\},\{\widetilde{4}\},\{\widetilde{5}\},\{6\}\}$. Actually, we will argue that the first kind of sub-currents only have $\tau^{0}$ order term, i.e., $J^{(0)}\left(D_{j}\right)=J\left(D_{j}\right)$. Nevertheless, we always use $J^{(0)}\left(D_{j}\right)$ in this paper to emphasize that there is no $\tau$ dependence in the final expression eq. (1.4). The behavior eq. (1.4) will be proved by Berends-Giele recursion.

We further generalize the off-shell recursion proofs of behaviors $a$ ) and b) to amplitudes containing more than one soft blocks and obtain the following results:

c) The $\tau^{0}$ term of an amplitude containing nonadjacent soft blocks, if one of which is of odd number soft pions, vanishes.

d) The $\tau^{0}$ term of an amplitude with at least four hard pions and nonadjacent soft blocks, where all blocks contain even number of soft pions, is a product of soft factors $\mathbb{S}^{(0)}$ for each block.

\footnotetext{
${ }^{3}$ The special case with only two hard pions, no matter they are adjacent or not, should have vanishing $\tau^{0}$ order.
} 
This paper is organized as follows. In section 2, we review off-shell and on-shell recursion relations for tree amplitudes in NLSM. In section 3, we illustrate that the leading order of partial amplitudes with adjacent soft pions of odd number is always vanishing. Behaviors of partial amplitudes with even number adjacent soft pions are studied section 4 . In section 5, we generalize our discussions to amplitudes with nonadjacent soft blocks. In section 6 , we conclude this work and discuss the physical insight of the multi-soft pions. We leave a tedious proof for a case of even number soft pion in the appendix as well as an explicit result of six soft pions.

\section{A review of off-shell and on-shell recursions in NLSM}

In this section, we briefly review both the off-shell Berends-Giele recursion in Cayley parameterization and on-shell recursion in NLSM, which will be used to prove multi-soft behaviors in this paper.

\subsection{Feynman rules and Berends-Giele recursion relations}

The on-shell amplitudes for SU( $N)$ NLSM are shown to be same with those in $\mathrm{U}(N)$ NLSM, thus we can just consider amplitudes in $\mathrm{U}(N)$ NLSM. Tree amplitudes of U( $N)$ NLSM can be decomposed into group structure factors and kinematic part as follows

$$
M\left(1^{a_{1}}, \ldots, n^{a_{n}}\right)=\sum_{\sigma \in S_{n-1}} \operatorname{Tr}\left(T^{a_{1}} T^{a_{\sigma_{2}}} \ldots T^{a_{\sigma_{n}}}\right) A(1, \sigma) .
$$

Due to the analogy between the group structure in NLSM and the color factors in YangMills theory, in the following discussion, the group structure factors in NLSM are mentioned as factors as well, and the kinematic part $A(1, \sigma)$ are color-ordered amplitudes which are evaluated by Feynman rules in Cayley parametrization, i.e.

$$
V_{2 n+1}=0, \quad V_{2 n+2}=\left(-\frac{1}{2 F^{2}}\right)^{n}\left(\sum_{i=0}^{n} k_{2 i+1}\right)^{2}=\left(-\frac{1}{2 F^{2}}\right)^{n}\left(\sum_{i=0}^{n} k_{2 i+2}\right)^{2} .
$$

Here, $k_{j}$ is the momentum of the external particle $j$ and note that the momentum conservation should be preserved. Apparently, in Cayley parametrization, only the scattering amplitudes with even number of external pions are nonzero, which of course is a fact independent of field definitions. Thus in this paper, we always consider pion amplitudes of even number external lines.

With Feynman rules eq. (2.2) at hand, tree-level currents can be constructed through Berends-Giele recursion relations (see [21, 22])

$$
\begin{aligned}
& J(2, \ldots, 2 n) \\
& =\frac{i}{P_{2,2 n}^{2}} \sum_{m=2}^{n} \sum_{\text {Divisions }} i V_{2 m}\left(k_{1}=-P_{2,2 n}, P_{A_{1}}, \cdots, P_{A_{2 m-1}}\right) \times \prod_{k=1}^{2 m-1} J\left(A_{k}\right),
\end{aligned}
$$

where $k_{1}=-P_{2,2 n}=-\left(k_{2}+k_{3}+\cdots+k_{2 n}\right)$ is the momentum of the off-shell pion " 1 ". In the second sum, "Divisions" denotes all possible partitions of on-shell particles $\{2, \ldots, 2 n\} \rightarrow$ 
$\left\{A_{1}\right\}, \ldots,\left\{A_{2 m-1}\right\}$ with odd number elements in each subset $\left\{A_{i}\right\}$. The starting point of this off-shell recursion is $J(2)=J(3)=\cdots=J(n)=1$. While multiplied by $-k_{1}^{2} \rightarrow 0$, the current (see eq. (2.3)) becomes on-shell amplitude $A(1, \cdots, n)$.

\subsection{On-shell recursion relations of effective theories}

In [23], on-shell recursion relations are proposed for effective theories of scalars. In particular, those amplitudes with vanishing single soft limits can be calculated by employing new rescaling momentum shifts, which works in NLSM, Dirac-Born-Infeld and Galileon theories. Now let us review the on-shell recursion for NLSM.

For $2 n$-point partial amplitude $A(1, \cdots, 2 n)$ in NLSM, we deform the momenta of all external pions as

$$
k_{\hat{i}}=\left(1-a_{i} z\right) k_{i} \quad(1 \leq i \leq 2 n) .
$$

The $2 n$-point partial amplitude $A_{n}(1, \cdots, 2 n)$ can be constructed from lower-point ones

$$
A(1, \cdots, 2 n)=\sum_{I} \frac{1}{P_{I}^{2}} \frac{A_{L}\left(z_{I}^{-}\right) A_{R}\left(z_{I}^{-}\right)}{\left(1-z_{I}^{-} / z_{I}^{+}\right) F_{2 n}\left(z_{I}^{-}\right)}+\left(z_{I}^{-} \leftrightarrow z_{I}^{+}\right),
$$

where all possible factorized channels $I$ are summed and the order of external pions are preserved. Given a partition $I, P_{I}=\sum_{i \in I} k_{i}$ and $z_{I}^{ \pm}$are the solutions to the on-shell condition of the internal propagator

$$
0=\left(P_{I}-z \sum_{i \in I} a_{i} k_{i}\right)^{2}=P_{I}^{2}-2 z\left(\sum_{i \in I} a_{i} k_{i}\right) \cdot P_{I}+z^{2}\left(\sum_{i \in I} a_{i} k_{i}\right)^{2},
$$

which results in the solutions

$$
z_{I}^{ \pm}=\frac{\left(\sum_{i \in I} a_{i} k_{i}\right) \cdot P_{I} \pm \sqrt{\left[\left(\sum_{i \in I} a_{i} k_{i}\right) \cdot P_{I}\right]^{2}-P_{I}^{2}\left(\sum_{i \in I} a_{i} k_{i}\right)^{2}}}{\left(\sum_{i \in I} a_{i} k_{i}\right)^{2}} .
$$

The function $F_{n}(z)$ in eq. (2.5) is defined by

$$
F_{2 n}(z)=\prod_{i=1}^{2 n}\left(1-a_{i} z\right)
$$

\section{Amplitudes with adjacent soft pions of odd number}

The vanishing of $\tau^{0}$ order with a single soft pion is already well known as Adler's zero. In this section, we generalize the Adler's zero to amplitudes with odd number of all-adjacent soft pions. Using both on-shell and off-shell recursion relations, we illustrate that the $\tau^{0}$ order of color-ordered tree amplitudes which containing arbitrary odd number of alladjacent soft pions has to vanish. 


\subsection{On-shell recursion approach}

Now let us study amplitudes with odd number of all-adjacent soft pions whose momenta are denoted by $\tau k_{i \in\{S\}}$, using the on-shell recursion reviewed in section 2.2.

First of all, the starting point of on-shell recursion approach is that four-point amplitude with one or three soft pions should vanish at $\tau^{0}$ order, which can be checked from direct evaluation by the Feyman rules eq. (2.2). One may notice that, according to momentum conservation, when three pions in a four-point amplitude are soft, the fourth one have to be soft too. If the momenta of all pions are of $\tau$ order, it seems meaningless to discuss soft limits any more. However, we are not really discussing about $2 n-1$ soft limits of $2 n$-point amplitude $A(1, \ldots, 2 n)$, and this special case only plays a role in the subamplitudes while applying on-shell recursion relations. Thus, the maximal odd number which makes the soft limits of $A(1, \ldots, 2 n)$ interesting and meaningful is $2 n-3$.

Assuming that the $\tau^{0}$ order of all $2 m(2 m<2 n)$-point amplitudes with odd number of soft pions vanish, we now use the on-shell recursion eq. (2.5) to investigate the behavior of $2 n$-point amplitude. In the on-shell recursion expression eq. (2.5) of $A(1, \ldots, 2 n)$, subamplitudes $A_{L}$ and $A_{R}$ contain at least four on-shell pions respectively: one comes from taking the internal line on-shell and others are external pions. According to different distributions of the $2 i+1$ soft pions, we classify terms in the recursion expression eq. (2.5) as follows.

(a) One subamplitude contains only soft external pions, while the other one contains hard external and probably soft pions.

(b) One subamplitude contains only hard external pions, while the other one contains both soft and at least two hard external pions.

(c) Both subamplitudes contain hard and soft pions.

In all three cases, the solution (2.7) can only start from $\tau^{0}$. This is because the leading behaviors of denominator and numerator of eq. (2.7) should be in a same order. Thus we only need to discuss the behaviors of subamplitudes $A_{L}, A_{R}$ and propagator $\frac{1}{P_{I}^{2}}$.

For the terms in category (a), without loss of generality, consider the case where $A_{L}$ contains only soft external pions, while $A_{R}$ contains both soft and hard external pions. Since all external pions in $A_{L}$ are soft, each of which contributes a factor $\tau$ in $A_{L}$ from its momentum. It pays to discuss the dimension and scale analysis about the amplitudes. In the NLSM with two derivatives, no matter what kind of parameterization is implemented, the vertex is of $p^{2}$ mass dimension order, the same as propagators. At classical level, the number of vertices $N_{\text {vertices }}$ and the number of propagators $N_{\text {propagators }}$ obeys $N_{\text {vertices }}=N_{\text {propagators }}+1$. Thus mass dimension of amplitudes should be $p^{2}$ order compensated by decay constant $F^{2}$. All soft external legs in $A_{L}$ should make the amplitude of $\tau^{2}$ order. The $\frac{1}{P_{I}^{2}}$ in eq. (2.5) contributes a $\frac{1}{\tau^{2}}$. In $A_{R}$, there is at least one soft pion, which comes from the internal propagator, and probably other even number soft external pions. In total, $A_{R}$ contains odd number of soft pions. According to the inductive assumption, the leading order of $A_{R}$ in soft limit is $\mathcal{O}(\tau)$. All together, terms of in category (a) obey a soft behavior of $\mathcal{O}(\tau)$. 
For the terms in category (b), the subamplitude $A_{L}$ containing only hard pions and $\frac{1}{P_{I}^{2}}$ are both of the order $\tau^{0}$, while the subamplitude $A_{R}$ contains odd number of soft pions thus should be of order $\mathcal{O}(\tau)$ from the inductive assumption.

For the terms in category (c), assume $A_{L}$ contains soft pions of odd number while $A_{R}$ contains soft pions of even number. The subamplitudes $A_{R}$ with even number all-adjacent soft pions, which will be systematically discussed in the next section, start from $\tau^{0}$ order. Remembering that the propagator $\frac{1}{P^{2}}$ also starts from $\tau^{0}$ order and the $\tau^{0}$ order of $A_{L}$ with adjacent odd number soft pions should vanish, we conclude that terms of this category must be zero. A very special case in this category is that $A_{R}$ contains only one hard external pion. In this case, the $\frac{1}{P_{I}^{2}}$ starts from $\mathcal{O}\left(\tau^{-1}\right), A_{R}$ is a subamplitude with soft pions of even number and two hard legs (one is the internal propagator), which behaves as $\mathcal{O}(\tau)$ according to discussion in the next section. The other subamplitude $A_{L}$, containing soft pions of odd number, behaves as $\mathcal{O}(\tau)$. Thus, terms of this category also behaves as $\mathcal{O}\left(\tau^{1}\right)$ as we expect.

Putting (a), (b) and (c) together, the vanishing of $\tau^{0}$ order of amplitudes in odd-point soft limit is proved by on-shell recursions.

\subsection{Berends-Giele recursion approach}

As a cross check for the behavior of amplitudes with adjacent odd number soft pions from on-shell recursion and as a warm-up for discussions on the behavior with even number soft pions, we apply the Berends-Giele recursion to prove the vanishing of the $\tau^{0}$ order of amplitudes containing adjacent odd number soft pions.

Consider a Berends-Giele current $J(2, \cdots, 2 n)$ defined by eq. (2.3) in Cayley parametrization. If all on-shell pions in this current are soft, namely $J(\widetilde{2}, \widetilde{3}, \ldots, \widetilde{2 n})$, from Feynman rules in eq. (2.2), each vertex in this current contributes a $\tau^{2}$, while each propagator in eq. (2.3) contributes a $\tau^{-2}$. In a tree-level Berends-Giele current, the number of propagators and of vertices are the same. Thus the behavior of such current is of order $\tau^{0}$. Actually, after dividing out all the $\tau$ factors, the $\tau^{0}$ term of $J(\widetilde{2}, \widetilde{3}, \ldots, \widetilde{2 n})$ is just a current $J(2,3, \ldots, 2 n)$ with replacing the momenta $k_{1}, \ldots, k_{2 n}$ by $q_{1}, \ldots, q_{2 n}$. If there exit more than one hard pions, the Berends-Giele currents with odd number of soft pions are of the generic form $J(2 \cdots, a, \widetilde{a+1}, \cdots, a \widetilde{+2 i}+1, a+2 i+2, \cdots, 2 n)$. According to whether $a$ is odd or even, these currents can be classified into two types.

- Type-1: if $a=2 l-1$, there are even number of hard pions appear before/after the first/last soft pion. The Berends-Giele current of this type behaves as

$$
J(2, \ldots, 2 l-1, \widetilde{2 l}, \ldots, \widetilde{2 l+2} i, 2 l+2 i+1, \ldots, 2 n)=0+\mathcal{O}\left(\tau^{1}\right) .
$$

While $l=1$, the current becomes $J(\widetilde{2}, \ldots, \widetilde{2+2 i}, 2 l+2 i+1, \ldots, 2 n)$ where the first soft pion is adjacent to the off-shell leg (see [7] for discussion about $l=1, i=0$ ). 
- Type-2: if $a=2 l$, there are odd number of hard pions appear before/after the first/last soft pions. The Berends-Giele current of this type behaves as

$$
\begin{gathered}
J(2, \ldots, 2 l, \widetilde{2 l+1}, \ldots, 2 l \widetilde{+2 i}+1,2 l+2 i+2, \ldots, 2 n) \\
\quad=-\sum_{D} \prod_{i=1}^{N_{D}}\left(-\frac{1}{2 F^{2}}\right)^{\frac{N_{D}-1}{2}} J^{(0)}\left(D_{i}\right)+\mathcal{O}\left(\tau^{1}\right) .
\end{gathered}
$$

Here, we summed over all possible divisions $D$ of $2, \ldots, 2 l, \widetilde{2 l+1}, \ldots, 2 l \widetilde{+2 i}+1,2 l+$ $2 i+2, \ldots, 2 n$ such that all the hard pions $\{2, \ldots, 2 l\}$ and $\{2 l+2 i+2, \ldots, 2 n\}$ belong to the first and the last set respectively. $J^{(0)}\left(D_{i}\right)$ stands for the $\tau^{0}$ order of current $J\left(D_{i}\right)$.

The on-shell limit of the soft behavior eq. (3.1) apparently gives zero result for $\tau^{0}$ order. When we multiply $k_{1}^{2} \rightarrow 0$ to the second type of soft behavior eq. (3.2), we can also see the vanish of $\tau^{0}$ order of on-shell amplitudes. Thus eq. (3.1) and eq. (3.2) precisely produce the expected $\tau^{0}$ behavior of amplitudes with odd number of all-adjacent soft pions. Assuming that all type-1/type-2 currents with fewer external pions satisfy eq. (3.1)/eq. (3.2), let us prove the behaviors (3.1) and (3.2) by induction.

Proof of eq. (3.1). When we expand $J(2 \cdots, a, \widetilde{a+1}, \cdots, a \widetilde{a+2 i+1}, a+2 i+2, \cdots, 2 n)$ through Berends-Giele recursions, corresponding diagrams can be further classified to two types: i) soft pions living in more than one sub-current, see figure $1(\mathrm{~A})$; ii) all soft pions in a same sub-current, see figure $1(\mathrm{~B})$.

Eq. (3.1) gives the behavior when $a=2 l-1$. If there are soft pions of odd number in the ordered set $\left\{H_{I}, S_{1}\right\},{ }^{4}$ (or $\left.\left\{S_{2 M+1}, H_{I+1}\right\}\right)$ in figure 1 (A), the current $J\left(H_{I}, S_{1}\right)$ (or $\left.J\left(S_{2 M+1}, H_{I+1}\right)\right)$ must be type- 1 currents. Thus its $\tau^{0}$ order must vanish according to the inductive assumption.

If both sets $\left\{H_{I}, S_{1}\right\}$ and $\left\{S_{2 M+1}, H_{I+1}\right\}$ in figure 1 (A) contain soft pions of even number (and hard pions of odd number), $I$ should be even, i.e., $I=2 L$. The $\tau^{0}$ order of figure $1(\mathrm{~A})$ is

$$
\begin{aligned}
& i\left(-\frac{1}{2 F^{2}}\right)^{N+M-1} \frac{i\left(\sum_{i=1}^{L} P_{H_{2 i-1}}+\sum_{j=L+1}^{N} P_{H_{2 j}}\right)^{2}}{\left(\sum_{i=1}^{2 L} P_{H_{i}}+\sum_{i=2 L+1}^{2 N} P_{H_{i}}\right)^{2}} \\
& \quad \times \prod_{i=1}^{2 L-1} J\left(H_{i}\right) J^{(0)}\left(H_{2 L}, S_{1}\right) \prod_{j=2}^{2 M} J^{(0)}\left(S_{j}\right) J^{(0)}\left(S_{2 M+1}, H_{2 L+1}\right) \prod_{l=2 L+1}^{2 N} J\left(H_{l}\right)+\mathcal{O}\left(\tau^{1}\right) .
\end{aligned}
$$

On the other hand, we turn to figure 1 (B). Since only the currents with odd number of external pions are nonzero and we have odd number soft pions, the sets $\left\{H_{I}\right\}$ and $\left\{H_{I+1}\right\}$ in $J\left(H_{I}, S, H_{I+1}\right)$ should simultaneously contain odd (or even) number of hard pions. If

\footnotetext{
${ }^{4}$ Here we use $\{S\},\{H\}$ to denote ordered sets of soft and hard pions respectively, which are used throughout this paper.
} 


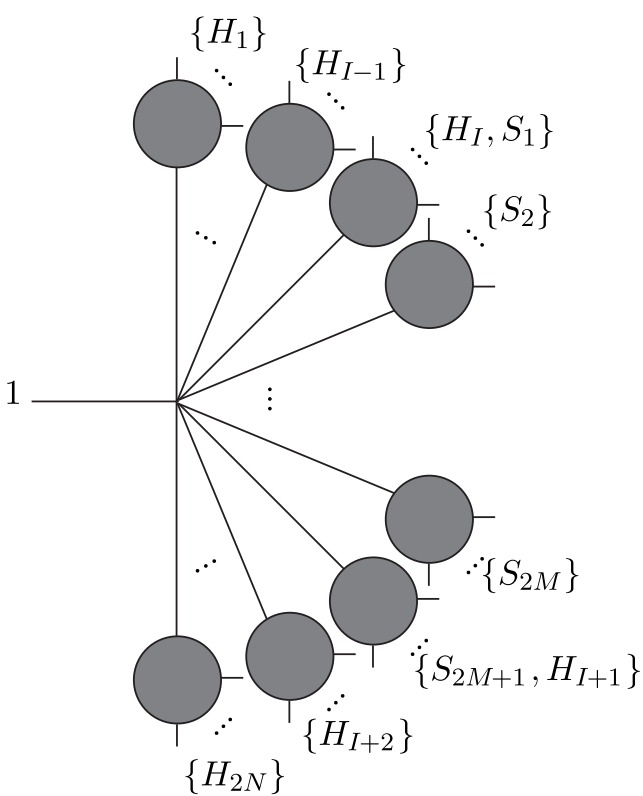

(A)

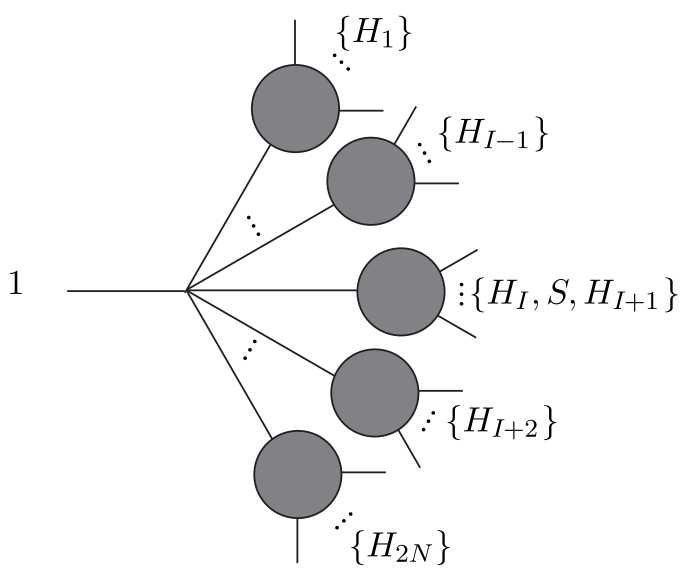

(B)

Figure 1. Typical diagrams for currents with odd number soft pions.

both $\left\{H_{I}\right\}$ and $\left\{H_{I+1}\right\}$ contain odd number of hard pions, the sub-current $J\left(H_{I}, S, H_{I+1}\right)$ have to vanish due to the inductive assumption. Therefore, $\left\{H_{I}\right\}$ and $\left\{H_{I+1}\right\}$ should both contain even number of hard pions. As a result, $I$ should be even, i.e., $I=2 L$ for some $L$. For a given figure $1(\mathrm{~A})$, there is always a corresponding figure $1(\mathrm{~B})$, whose leading order is following

$$
\begin{aligned}
& i\left(-\frac{1}{2 F^{2}}\right)^{N-1} \frac{i\left(\sum_{i=1}^{L} P_{H_{2 i-1}}+\sum_{j=L+1}^{N} P_{H_{2 j}}\right)^{2}}{\left(\sum_{i=1}^{2 L} P_{H_{i}}+\sum_{i=2 L+1}^{2 N} P_{H_{i}}\right)^{2}} \prod_{i=1}^{2 L-1} J\left(H_{i}\right) \prod_{l=2 L+1}^{2 N} J\left(H_{l}\right) J^{(0)}\left(H_{2 L}, S, H_{2 L+1}\right) \\
& \quad+\mathcal{O}\left(\tau^{1}\right) .
\end{aligned}
$$

Here, $J^{(0)}\left(H_{2 L}, S, H_{2 L+1}\right)$ is the leading order of sub-current $J\left(H_{2 L}, S, H_{2 L+1}\right)$ and can be further recursively written out by eq. (3.2). Apparently, there always exist one term in the expression of $J^{(0)}\left(H_{2 L}, S, H_{2 L+1}\right)$ :

$$
-\left(-\frac{1}{2 F^{2}}\right)^{M} J^{(0)}\left(H_{2 L}, S_{1}\right) \prod_{j=2}^{2 M} J^{(0)}\left(S_{j}\right) J^{(0)}\left(S_{2 M+1}, H_{2 L+1}\right),
$$

which together with eq. (3.4) produces eq. (3.3) with an opposite sign precisely. Thus such term from figure 1 (B) cancel with figure 1 (A) for given $\left\{H_{I}, S_{1}\right\},\left\{S_{2}\right\}, \cdots,\left\{S_{2 M}\right\}$, $\left\{S_{2 M+1}, H_{I+1}\right\}$. After considering all such cancelations, we arrive the expected behavior eq. (3.1). 
Proof of eq. (3.2). Eq. (3.2) gives the behavior with $a=2 l$. In this case, typical diagrams are also given by figure $1(\mathrm{~A})$ and (B) but with $a=2 l$. There are odd number of hard pions appear before/after the first/last soft pion. As already discussed in the proof of eq. (3.2), Only the figure $1(\mathrm{~A})$ and (B) with both $\left\{H_{I}\right\}$ and $\left\{H_{I+1}\right\}$ contain odd number hard pions provide nonzero contributions. Consequently, $I$ in figure $1(\mathrm{~A})$ and (B) should be odd, i.e., $I=2 L+1$ for some $L$.

For any diagram given by figure 1 (A) with $L>0$ or $L<N-1$, one can always find a corresponding cancelation from figure 1(B), as shown in the proof of eq. (3.1). A subtle situation comes from $L=0$ and $N=1$. In this case, all hard pions in figure 1 (A) appearing before/after the first/last soft pion belong to a single sub-current $J\left(H_{1}, S_{1}\right) / J\left(S_{2 M+1}, H_{2}\right)$. There is no corresponding figure 1 (B) can be found. For each figure 1 (A) of this special case, the leading orders of propagator and vertex are $\frac{i}{\left(P_{H_{1}}+P_{H_{2}}\right)^{2}}$ and $i\left(-\frac{1}{2 F^{2}}\right)^{M}\left(P_{H_{1}}+P_{H_{2}}\right)^{2}$, while the sub-currents contribute

$$
-\left(-\frac{1}{2 F^{2}}\right)^{M} J^{(0)}\left(H_{1}, S_{1}\right) \prod_{i=2}^{2 M} J^{(0)}\left(S_{i}\right) J^{(0)}\left(S_{2 M+1}, H_{2}\right),
$$

which is exactly one term in eq. (3.2). After summing all diagrams of this special case, we get the right hand side of eq. (3.2).

\section{Amplitudes with adjacent soft pions of even number}

Now let us consider the behavior of amplitude $A(1, \cdots, a, \widetilde{a+1}, \cdots, \widetilde{a+2} i, a+2 i+1, \cdots, 2 n)$ with adjacent soft pions of even number and prove eq. (1.3). If the amplitude contains two hard pions, one can use both on-shell recursion and off-shell Berends-Giele recursion directly to prove that the $\tau^{0}$ order should vanish. In this section, we consider the nontrivial case with at least four hard pions. First of all, we prove the adjacent even soft limits of Berends-Giele currents (defined by eq. (2.3)) instead of amplitudes inductively. With the currents' results at hand, the amplitude version is derived eventually, as eq. (1.3) in the introduction. Since we will use Berends-Giele recursion relations in the following discussion, different relative positions between the soft pions and the off-shell line should be considered.

\subsection{One soft pion is adjacent to the off-shell line}

If one soft pion is adjacent to the off-shell line, the Berends-Giele current with soft pions of even number has the form $J(\widetilde{2}, \ldots, \widetilde{2 i+1}, 2 i+2, \ldots, 2 n)$. When $i=n-1$, the current only contain one hard pion, one can easily check that this current is of $\tau^{0}$ order for $n=2$ and prove that $J(\widetilde{2}, \ldots, \widetilde{2 n-1}, 2 n)$ is also of order $\tau^{0}$ by Berends-Giele recursion for $n>2$. If $i<n-1$, the current $J(\widetilde{2}, \ldots, \widetilde{2 i+1}, 2 i+2, \ldots, 2 n)$ in the soft limit $\tau \rightarrow 0$ should obey the following identity

$$
J(\widetilde{2}, \cdots, \widetilde{2 i+1}, 2 i+2, \cdots, 2 n)=J^{(0)}(\widetilde{2}, \cdots, \widetilde{2 i+1}, 2 i+2) J(2 i+2, \cdots, 2 n),
$$

where $J^{(0)}(\widetilde{2}, \cdots, \widetilde{2 i+1}, 2 i+2)$ is the $\tau^{0}$ order term of $J(\widetilde{2}, \cdots, \widetilde{2 i+1}, 2 i+2)$. To prove this identity, we classify the diagrams in Berends-Giele recursion eq. (2.3) with a criterion that all soft pions are in a single sub-current or not. 


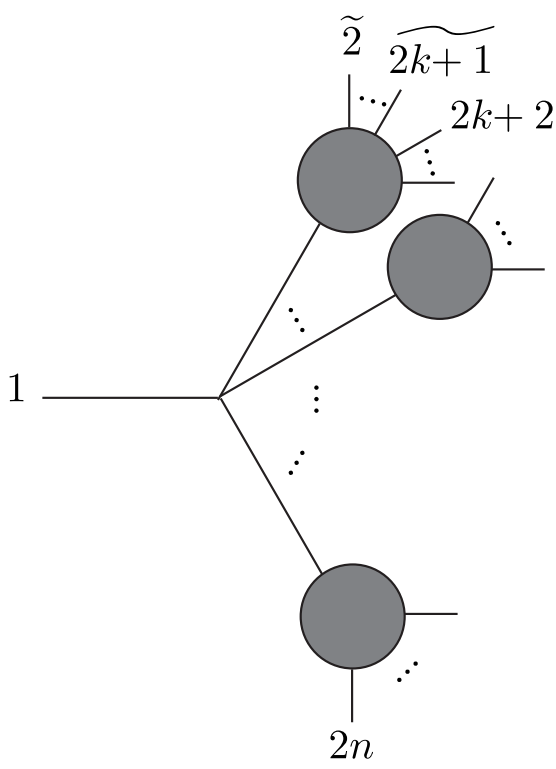

(A)

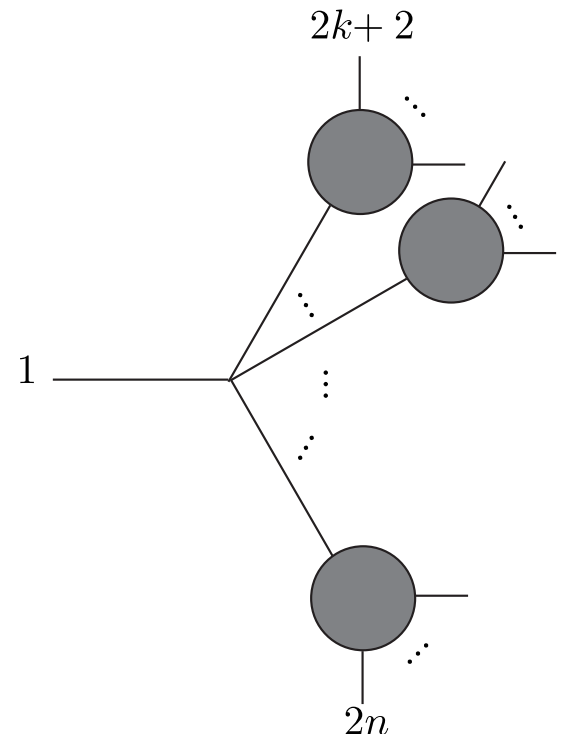

(B)

Figure 2. (A) is a typical diagram for $J(\widetilde{2}, \cdots, \widetilde{2 i+1}, 2 i+2, \cdots)$ with all soft pions living in a same sub-current. When the soft factor $J^{(0)}(\widetilde{2}, \cdots, \widetilde{2 k+2}, 2 k+2)$ is extracted, the diagram (A) becomes diagram (B).

\section{- All soft pions are in a single sub-current}

A typical diagram where all soft pions are located in a single sub-current is expressed in figure 2(A). Since a sub-current can only contain odd number of external pions, there must be even number soft pions and odd number hard pions in the first sub-current. From inductive assumption, the sub-current $J(\widetilde{2}, \cdots, \widetilde{2 i+1}, 2 i+2, \cdots)$ satisfies eq. (4.1) when $\tau \rightarrow 0$. When the leading soft factor $J^{(0)}(\widetilde{2}, \ldots, \widetilde{2 i+1}, 2 i+2, \cdots)$ is extracted out, figure $2(\mathrm{~A})$ becomes figure $2(\mathrm{~B})$. Thus we established a one to one correspondence between diagrams (A) and (B) in figure 2. Notice that the summation of all diagrams (B) is exactly the Berends-Giele current of only hard pions, i.e., $J(2 i+2, \cdots, 2 n)$. All together, the collection of this type diagrams with all soft pions in a single sub-current result in eq. (4.1).

\section{- More than one sub-currents contain soft pions}

A typical diagram of this type is given by figure $3(\mathrm{~A})$. The ordered set $\left\{S_{I}, H_{1}\right\}$ in figure $3(\mathrm{~A})$ contains both soft and hard external pions. If there are odd number of soft pions in $\left\{S_{I}, H_{1}\right\}$, the $\tau^{0}$ order of the sub-current $J\left(S_{I}, H_{1}\right)$ is zero as already stated in section 3.2. Thus $\left\{S_{I}, H_{1}\right\}$ can only contain soft pions of even number. The boundary case of figure $3(\mathrm{~A})$, where $S_{I}$ is empty and $\left\{S_{I}, H_{1}\right\}=\left\{H_{I}\right\}$ only consists of hard pions, must also be taken into account. The $\tau^{0}(\tau \rightarrow 0)$ order of figure $3(\mathrm{~A})$ evaluates (for convenience 


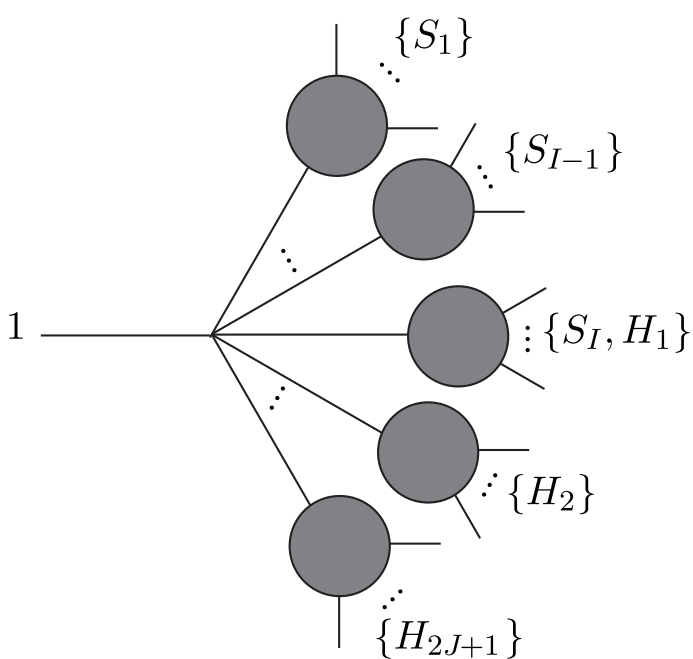

(A)

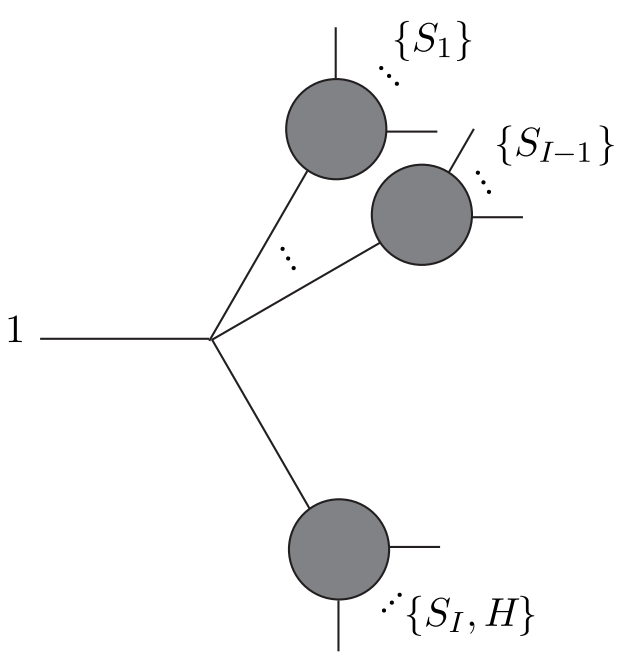

(B)

Figure 3. Tpypical diagrams for $J(\widetilde{2}, \cdots, \widetilde{2 i+1}, 2 i+2, \cdots)$ with soft pions belong to more than one sub-currents: (A) is a diagram with hard pions belong to more than one sub-current; (B) is a diagram with all hard pions belong to a same sub-current.

we omit the coupling constants which do not affect our discussions)

$$
\left.\frac{i}{\left(P_{H_{1}}+P_{H_{2}}+\cdots+P_{H_{2 J+1}}\right)^{2}} i\left(\sum_{l=0}^{J} P_{H_{2 l+1}}\right)^{2}\left[\prod_{l=1}^{I-1} J^{(0)}\left(S_{i}\right)\right] J\left(S_{I}, H_{1}\right)\right|_{\tau \rightarrow 0} \prod_{l=2}^{2 J+1} J\left(H_{l}\right) .
$$

From inductive assumption, we can use eq. (4.1) to express $\left.J\left(S_{I}, H_{1}\right)\right|_{\tau \rightarrow 0}$ as

$$
\left.J\left(S_{I}, H_{1}\right)\right|_{\tau \rightarrow 0}=J^{(0)}\left(S_{I}, 2 k+2\right) J\left(H_{1}\right)
$$

where $2 k+2$ is the first hard pion in $\left\{H_{1}\right\}$. Thus the leading order of figure $3(\mathrm{~A})$ behaves as

$$
\frac{i}{\left(P_{H_{1}}+P_{H_{2}}+\cdots+P_{H_{2 J+1}}\right)^{2}} i\left(\sum_{l=0}^{J} P_{H_{2 l+1}}\right)^{2}\left[\prod_{l=1}^{I-1} J^{(0)}\left(S_{i}\right)\right] J^{(0)}\left(S_{I}, 2 k+2\right) \prod_{l=1}^{2 J+1} J\left(H_{l}\right) \text {. }
$$

The special case with $J=0$, where all hard pions belong to a single sub-current as shown by figure $3(\mathrm{~B})$, should be studied separately. One can find cancellations between diagrams (A) and (B) in figure 3. To see this, we compute the leading behavior of the special case figure 3 (B), with eq. (4.1) for lower-point sub-current and obtain

$$
\frac{i}{\left(P_{H_{1}}+\cdots+P_{H_{2 J+1}}\right)^{2}} i\left(\sum_{j=1}^{2 J+1} P_{H_{j}}\right)^{2}\left[\prod_{l=1}^{I-1} J^{(0)}\left(S_{i}\right)\right] J^{(0)}\left(S_{I}, 2 k+2\right) J(2 k+2, \cdots, 2 n) .
$$


According to Berends-Giele recursion eq. (2.3), the current $J(2 k+2, \cdots, 2 n)$ can be expanded as

$$
J(2 k+2, \cdots, 2 n)=\frac{i}{\left(\sum_{j=1}^{2 J+1} P_{H_{j}}\right)^{2}} \sum_{\text {Divisions }} i\left(\sum_{l=0}^{J} P_{H_{2 l+1}}\right)^{2} \prod_{l=1}^{2 J+1} J\left(H_{l}\right) .
$$

Here we summed over all possible divisions of hard pions $\{2 k+2, \ldots, 2 n\} \rightarrow\left\{H_{1}\right\}, \cdots$, $\left\{H_{2 J+1}\right\}$, where each subset contains odd number of hard pions. Apparently, eq. (4.4) always cancels with the corresponding division $\left\{H_{1}\right\}, \cdots,\left\{H_{2 J+1}\right\}$ of eq. (4.5), thus this type of diagrams do not contribute at all.

Summing all two types of diagrams, the proof of the leading behavior eq. (4.1) of current with one soft pion adjacent to the off-shell line is completed. This conclusion will be used as a known fact in our following discussion about the situation that no soft pion is adjacent to the off-shell leg.

\subsection{All soft pions are nonadjacent to the off-shell line}

Now let us study the behavior of Berends-Giele current $J(1, \cdots, a, \widetilde{a+1}, \cdots, \widetilde{a+2 i}$, $a+2 i+1, \cdots, 2 n)$ where no soft pion is adjacent to the off-shell leg and show that

$$
\begin{aligned}
& J(2, \cdots, a, \widetilde{a+1}, \cdots, \widetilde{a+2} i, a+2 i+1, \cdots, 2 n) \\
& \quad=\mathbb{S}_{a, a+2 i+1}^{(0)} J(1, \cdots, a, a+2 i+1, \cdots, 2 n)+\mathcal{O}(\tau) .
\end{aligned}
$$

Here the soft factor $\mathbb{S}_{a, a+2 i+1}^{(0)}$ is defined by eq. (1.4).

To prove the above behavior, we expand $J(2, \cdots, a, \widetilde{a+1}, \cdots, \widetilde{a+2} i, a+2 i+1, \cdots, 2 n)$ by Berends-Giele recursion. According to whether the two hard pions $a$ and $a+2 i+1$ are in a same sub-current or not, the diagrams in Berends-Giele recursion expressions are classified into two types, shown by figure 4 (A) and (B) respectively. Substituting eq. (4.7) into the sub-current $J\left(H_{L}, S, H_{R}\right)$ and extracting the soft factor $\mathbb{S}_{a, a+2 i+1}^{(0)}$ out, we find that figure $4(\mathrm{~A})$ becomes a typical diagram of the Berends-Giele recursion expression of $J(1, \cdots, a, a+2 i+1, \cdots, 2 n)$, where $a, a+2 i+1$ are in a same sub-current.

On the other hand, when eq. (4.1) is applied to sub-currents $J\left(H_{I}, S_{1}\right)$ and $J\left(S_{K}, H_{I+1}\right)$, the leading behavior of figure 4 (B) can be written as

$J^{(0)}\left(a, S_{1}\right) J^{(0)}\left(S_{2}\right) \cdots J^{(0)}\left(S_{K}, a+2 i+1\right)\left[\frac{i}{\left(P_{H_{1}}+\cdots+P_{H_{2 J+1}}\right)^{2}} i\left(\sum_{l=0}^{J} P_{H_{2 l+1}}\right)^{2} \prod_{b=1}^{2 J+1} J\left(H_{j}\right)\right]$.

For diangrams shown by figure $4(\mathrm{~B})$ with given division of hard pions $\left\{H_{1}\right\}, \cdots,\left\{H_{2 J+1}\right\}$, once summing over all possible $J^{(0)}\left(a, S_{1}\right) J^{(0)}\left(S_{2}\right) \cdots J^{(0)}\left(S_{K}, a+2 i+1\right)$, one can get a diagram, in which all external lines are hard pions and the pions $a, a+2 i+1$ are separated in two different sub-currents, multiplied by a soft factor eq. (1.4).

To conclude, up to a soft factor eq. (1.4), the (A) type of diagrams produce diagrams in $J(1, \cdots, a, a+2 i+1, \cdots, 2 n)$ with $a, a+2 i+1$ in a same sub-currents while the (B) type 


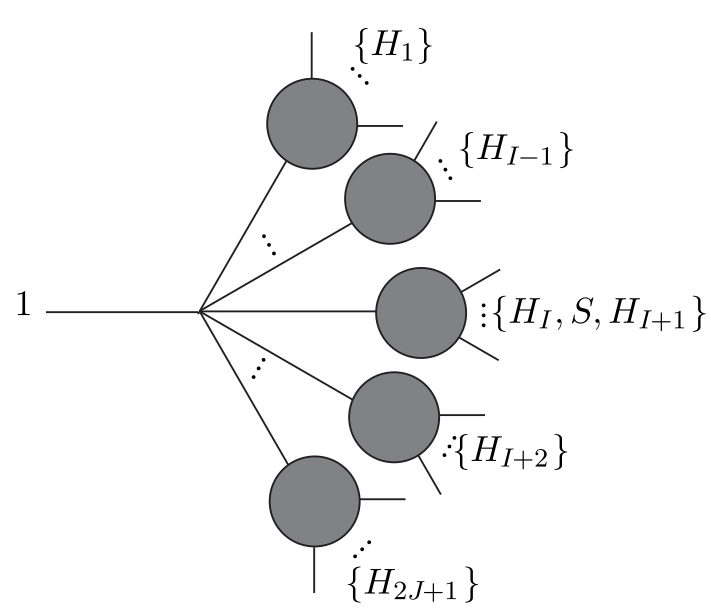

(A)

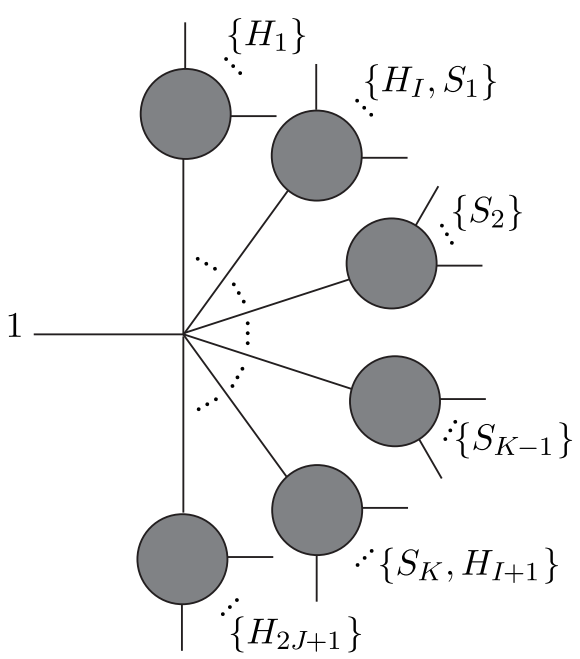

(B)

Figure 4. Typical diagrams of $J(2, \cdots, a, \widetilde{a+1}, \cdots, \widetilde{a+2 i}, a+2 i+1, \cdots, 2 n)$ : (A) is a diagram with all soft pions living in a same sub-current; (B) is a diagram with soft pions belong to more than one diagram.

of diagrams produce those diagrams in $J(1, \cdots, a, a+2 i+1, \cdots, 2 n)$ with $a, a+2 i+1$ in two different sub-currents. Summing all possible (A) and (B) types of diagrams in figure 4 together and putting coupling constants back, we arrive eq. (4.7).

\subsection{The behavior of on-shell amplitudes with even number of adjacent soft pions}

Now we are ready to take the on-shell limit. When $-P_{2,2 n}^{2}$ is multiplied to the leading term of the currents $J(2, \cdots, a, \widetilde{a+1}, \cdots, \widetilde{a+2 i}, a+2 i+1, \cdots, 2 n)$ in eq. (4.7) with no soft pion adjacent to the off-shell line, we get $\mathbb{S}_{a+1, a+2 i}^{(0)} A(1, \cdots, a, a+2 i+1, \cdots, 2 n)$, which is the expected leading order multi-soft behavior eq. (1.3).

The on-shell limit of currents with one soft pion adjacent to the off-shell line is more subtle. Typical diagrams of this case are shown by figure $5(\mathrm{~A})$ and (B). Apparently, if $-P_{2,2 n}^{2}$ is multiplied to eq. (4.1) directly, the expected behavior eq. (1.3) of on-shell amplitudes can not be obtained. This is because when we consider the on-shell limit $k_{1} \rightarrow 0$ of figure $5(\mathrm{~B})$, the propagator of the off-shell leg in the sub-current $J\left(S_{2 I+1}, H\right)$, which contain all hard pions in it, should be divergent in the soft limit $\tau \rightarrow 0$. In the appendix A, the semi-amplitude $A^{*}\left(1^{*}, 2, \ldots, 2 n\right) \equiv-P_{2,2 n}^{2} J(2, \ldots, 2 n)$ instead of BerendsGiele current is used to study the multi-soft behavior in this case. This semi-amplitude is nothing but the sum of all possible Feyman diagrams where $2, \cdots, 2 n$ are on-shell. We will prove that a semi-amplitude $A^{*}\left(k_{1}+\tau \sum_{j=2}^{2 m-1} q_{j}, \widetilde{2 m}, \widetilde{2 m+1}, \cdots, \widetilde{2 i+1}, 2 i+2 \cdots, 2 n\right)$ obeys the following identity

$$
\begin{aligned}
A^{*} & \left(k_{1}+\tau \sum_{j=2}^{2 m-1} q_{j}, \widetilde{2 m}, \widetilde{2 m+1}, \cdots, \widetilde{2 i+1}, 2 i+2 \cdots, 2 n\right) \\
= & \mathbb{S}^{*(0)} k_{1}+\tau \sum_{j=2}^{2 m-1} q_{j}, 2 i+2
\end{aligned}
$$




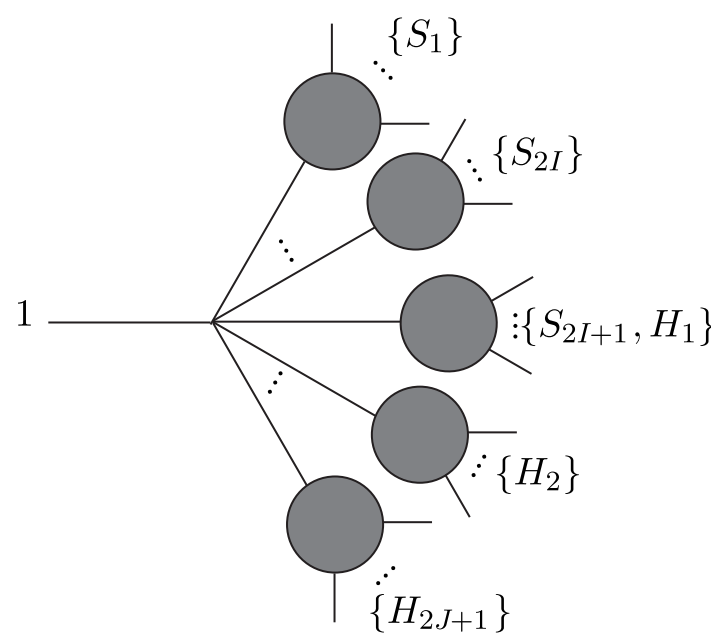

(A)

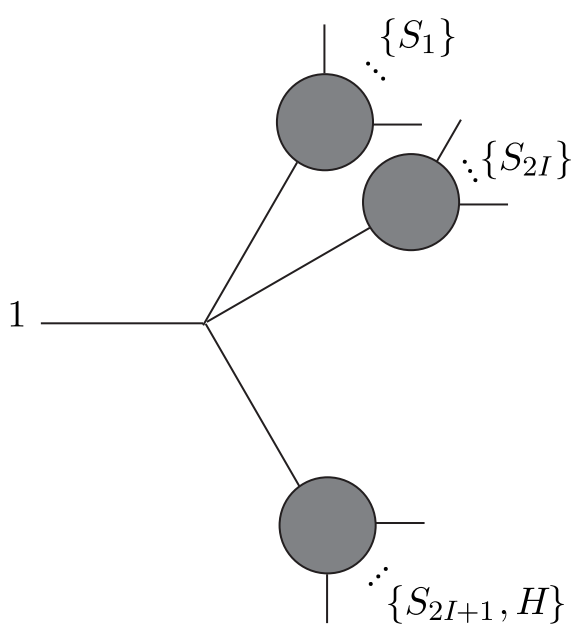

(B)

Figure 5. When the pions 1 is set to on-shell, there are two types of diagrams for the amplitude $A(1, \widetilde{2}, \cdots, \widetilde{2 i+1}, 2 i+2, \cdots, 2 n)$ : (A) stands for diagrams with hard pions belong to different sub-currents; (B) stands for diagrams with all hard pions living in a same sub-current.

Here we use a star $^{*}$ to emphasize that the semi-amplitude and soft factors, which are expressed by products of $\tau^{0}$ order of sub-currents, are extended to off-shell case by just allowing external off-shell legs in the Feynman rules eq. (2.2). When $m=1$, the momentum of the first leg becomes the on-shell momentum $k_{1}$, we get the expected soft behavior of amplitude $A(1, \widetilde{2}, \cdots, \widetilde{2 i+1}, 2 i+2, \cdots, 2 n)=\mathbb{S}_{1,2 i+2}^{(0)} A(1,2 i+2, \cdots, 2 n)+\mathcal{O}(\tau)$. Details can be found in appendix A.

\subsection{Quadruple soft limits}

When we have two adjacent soft pions, the behavior eq. (1.3), having three possible divisions in the soft factor eq. (1.3), precisely reproduces the double soft behavior [3, 6-8, 22]. let us have a look at the explicit expression of quadruple soft limit with adjacent soft pions. By

substituting the explicit expression of $J^{(0)}$ 's corresponding to the divisions eq. (1.5) into the soft factor eq. (1.4) with four adjacent soft pions, we obtain

$$
\begin{aligned}
A_{8}(1, \widetilde{2}, \widetilde{3}, \widetilde{4}, \widetilde{5}, 6, \cdots, 2 n) \\
=\frac{1}{4 F^{4}} A_{4}(1,6, \cdots, 2 n)\left[-\frac{s_{2,4}}{s_{234}} \frac{q_{5} \cdot k_{6}}{\left(q_{2}+q_{3}+q_{4}+q_{5}\right) \cdot k_{6}}+\frac{s_{2,4}}{s_{234}} \frac{q_{4} \cdot k_{1}}{\left(q_{2}+q_{3}+q_{4}+q_{5}\right) \cdot k_{1}}\right. \\
\quad+\frac{s_{3,5}}{s_{345}} \frac{q_{2} \cdot k_{6}}{\left(q_{2}+q_{3}+q_{4}+q_{5}\right) \cdot k_{6}}-\frac{s_{3,5}}{s_{345}} \frac{q_{2} \cdot k_{1}}{\left(q_{2}+q_{3}+q_{4}+q_{5}\right) \cdot k_{1}} \\
\quad-\frac{q_{2} \cdot k_{6}}{\left(q_{2}+q_{3}+q_{4}+q_{5}\right) \cdot k_{6}} \frac{q_{5} \cdot k_{6}}{\left(q_{4}+q_{5}\right) \cdot k_{6}}-\frac{q_{2} \cdot k_{1}}{\left(q_{2}+q_{3}\right) \cdot k_{1}} \frac{q_{5} \cdot k_{1}}{\left(q_{1}+q_{2}+q_{3}+q_{4}\right) \cdot k_{1}} \\
\left.\quad+\frac{q_{5} \cdot k_{6}}{\left(q_{4}+q_{5}\right) \cdot k_{6}} \frac{q_{2} \cdot k_{1}}{\left(q_{2}+q_{3}\right) \cdot k_{1}}\right] .
\end{aligned}
$$

The sextuple soft factor is also derived but with a long expression, we put it in appendix B. 


\section{$5 \quad$ Amplitudes with nonadjacent soft blocks}

In this section, we generalize the discussions in section 3 and section 4 to amplitudes containing nonadjacent soft blocks as

$$
\begin{gathered}
A\left(1, \cdots, a_{1},\left\{\widetilde{a_{1}+1}, \cdots, \widetilde{a_{1}+i_{1}}\right\}, a_{1}+i_{1}+1, \cdots, a_{2},\left\{\widetilde{a_{2}+1}, \cdots, \widetilde{a_{2}+i_{2}}\right\}, a_{2}+i_{2}+1,\right. \\
\left.\quad \cdots, a_{r},\left\{\widetilde{a_{r}+1}, \cdots, \widetilde{a_{r}+i_{r}}\right\}, a_{r}+i_{r}+1, \cdots\right) .
\end{gathered}
$$

- If at least one soft block, e.g., $\left\{\widetilde{a_{j}+1}, \cdots, \widetilde{a_{j}+i_{j}}\right\}(1 \leq j \leq r)$, contains soft pions of odd number, the $\tau^{0}$ order of amplitude must vanish. This can be directly seen when replacing some of the hard pions in $\left\{2, \cdots, a_{j}-1\right\}$ and $\left\{a_{j}+i_{j}+2, \cdots, 2 n\right\}$ of $J\left(2 \cdots, a_{j}, \widetilde{a_{j}+1}, \cdots, \widetilde{a_{j}+i_{j}}, a+i_{j}+1, \cdots, 2 n\right)$ by some soft ones and repeating the proof procedure in section 3 .

- If every soft block contains soft pions of even number, the proof given in section 4 can be applied. In particular, through a similar proof in section 4 , the following iterative expression for amplitudes with soft blocks of even order is obtained

$$
\begin{aligned}
& \left.A\left(1, \cdots, a_{1}, \widetilde{a_{1}+1}, \cdots, \widetilde{a_{1}+2 i_{1}}\right\}, a_{1}+2 i_{1}+1, \cdots, a_{2}, \widetilde{\left\{a_{2}+1\right.}, \cdots, \widetilde{a_{2}+2 i_{2}}\right\}, a_{2}+2 i_{2}+1, \\
& \left.\cdots, a_{r},\left\{\widetilde{a_{r}+1}, \cdots, \widetilde{a_{r}+2} i_{r}\right\}, a_{r}+2 i_{r}+1, \cdots\right) \\
& =\mathbb{S}_{a_{1}, a_{1}+2 i_{1}+1}^{(0)} A^{(0)}\left(\cdots, a_{2},\left\{\widetilde{a_{2}+1}, \cdots, \widetilde{a_{2}+2} i_{2}\right\}, a_{2}+2 i_{2}+1,\right. \\
& \left.\cdots, a_{r},\left\{\widetilde{a_{r}+1}, \cdots, \widetilde{a_{r}+2} i_{r}\right\}, a_{r}+2 i_{r}+1, \cdots\right)+\mathcal{O}(\tau) .
\end{aligned}
$$

The special case with only two hard pions and two even-number soft blocks, which has vanishing $\tau^{0}$ order behavior, is not included in the above discussion.

Repeating this iterative procedure, we finally get the leading behavior

$$
\begin{aligned}
& A\left(1, \cdots, a_{1},\left\{\widetilde{a_{1}+1}, \cdots, \widetilde{a_{1}+2 i_{1}}\right\}, a_{1}+2 i_{1}+1, \cdots, a_{2}, \widetilde{a_{2}+1}, \cdots, \widetilde{a_{2}+2 i_{2}}\right\}, a_{2}+2 i_{2}+1, \\
& \left.\quad \cdots, a_{r},\left\{\widetilde{a_{r}+1}, \cdots, \widetilde{a_{r}+2} i_{r}\right\}, a_{r}+2 i_{r}+1, \cdots\right) \\
& =\prod_{j=1}^{r} \mathbb{S}_{a_{j}, a_{j}+2 i_{j}+1}^{(0)} A\left(\cdots, a_{1}, a_{1}+2 i_{1}+1, \cdots, a_{2}, a_{2+2 i_{2}+1}, \cdots, a_{r}, a_{r}+2 i_{r}+1, \cdots\right)+\mathcal{O}(\tau) .
\end{aligned}
$$

This result is always the same no matter consecutively or simultaneously extracting soft factors.

\section{Conclusion}

In this paper, all leading order multi-soft behaviors of tree amplitudes in NLSM are discussed. We showed that the leading order behavior of an amplitude with odd number alladjacent soft pions vanishes, while the leading behavior of an amplitude with all-adjacent even number of soft pions is described by sum of products of $\tau^{0}$ order Berends-Giele subcurrents. Furthermore, we generalized our discussions to amplitudes with nonadjacent 
blocks of soft pions: the amplitudes containing at least one block with soft pions of odd number have to vanish at the leading order; if each block contains soft elements of even number, the leading order is given by products of soft factors for those blocks.

Several questions are worth further studying: a) How to explain the multi-soft factor, especially the relations with the commutations of the generators for the broken and unbroken symmetry; b) It would be interesting to have a look at the sub-leading order soft behavior, which might contain more insights about the global symmetry breaking; c) We only discussed global symmetry breaking in this paper, then multi-soft behavior of supersymmetry as well as conformal symmetry breaking is another interesting topic. d) It is worthy studying these soft behaviors of NLSM from other perspectives, e.g, CachazoHe-Yuan formula [25-29] and abelian Z-theory [30].

\section{Acknowledgments}

We would like to thank Rutger Boels, Clifford Cheung, Ian Low and Congkao Wen for helpful suggestions. YD would like to acknowledge National Natural Science Foundation of China under Grant Nos. 11105118, 111547310, as well as the 351 program of Wuhan University. HL is supported by the German Science Foundation (DFG) within the Collaborative Research Center 676 "Particles, Strings and the Early Universe".

\section{A The proof of eq. (4.9)}

\section{A.1 An example}

Consider an amplitude $A(1, \widetilde{2}, \widetilde{3}, \widetilde{4}, \widetilde{5}, \widetilde{6}, \widetilde{7}, 8,9,10)$ and choose the momentum of the pion 1 to be expressed through momentum conservation, in other words, leg 1 is chosen to be off-shell in the Berends-Giele recursions. While Berends-Giele recursions are applied to rewrite this amplitude, a type of sub-currents where all hard lines are included in a same sub-current emerges, e.g., the sub-current $J(\widetilde{4}, \widetilde{5}, \widetilde{6}, \widetilde{7}, 8,9,10)$. Since the propagator $\frac{i}{P_{4,10}^{2}}=$ $\frac{i}{\left(k_{1}+\tau q_{2}+\tau q_{3}\right)^{2}}$ behaves as $\frac{1}{\tau}$ when taking $\tau \rightarrow 0$, the sub-current seems to be divergent. Nevertheless, this divergence will disappear when we turn to the full amplitude. Thus, it's more convenient to consider a semi-amplitude $A^{*}\left(k_{1}+\tau q_{2}+\tau q_{3}, \widetilde{4}, \widetilde{5}, \widetilde{6}, \widetilde{7}, 8,9,10\right)=$ $\left(-P_{\left(k_{1}+\tau q_{2}+\tau q_{3}\right)}^{2}\right) J(\widetilde{4}, \widetilde{5}, \widetilde{6}, \widetilde{7}, 8,9,10)$ instead. This semi-amplitude consist of all possible Feynman diagrams, but with some of external leg are off-shell. For example the semiamplitude $A^{*}\left(k_{1}+\tau q_{2}+\tau q_{3}, \widetilde{4}, \widetilde{5}, \widetilde{6}, \widetilde{7}, 8,9,10\right)$, the first leg $k_{1}+\tau q_{2}+\tau q_{3}$ is not on-shell unless taking a soft limit. This semi-amplitude can be expanded through the Berends-Giele recursions and classified into the following two types of diagrams. ${ }^{5}$

- Diagrams where hard pions are included in more than one sub-currents (see figure 6).

Since diagrams figure $6(\mathrm{~B} 4),(\mathrm{C} 1)$ and (C3) contain sub-currents with odd number of adjacent soft pions, they must have vanishing $\tau^{0}$ order in the soft limits $\tau \rightarrow 0$. The

\footnotetext{
${ }^{5}$ Again, we omit coupling constants and put them back in the final expression.
} 


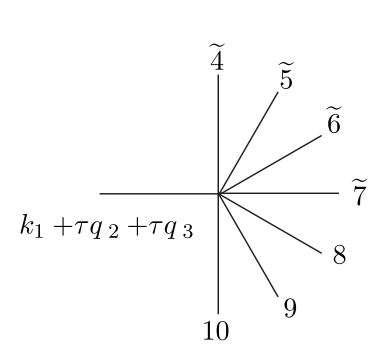

(A1)

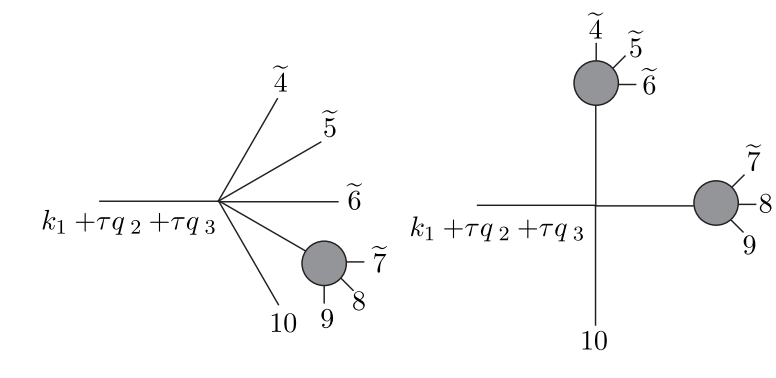

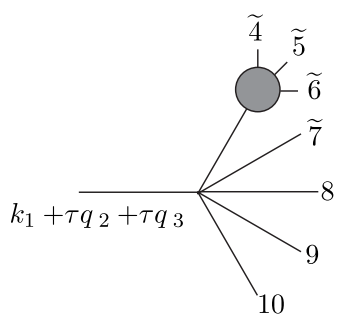

(B1)
(B4)

$(\mathrm{C} 1)$

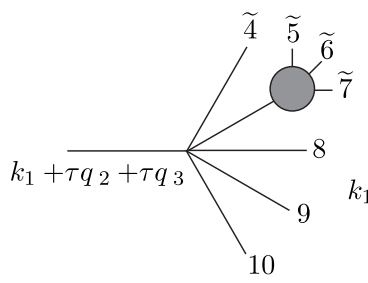

(B2)

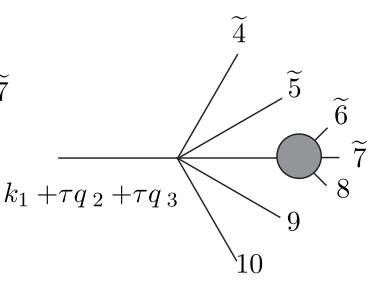

(B3)

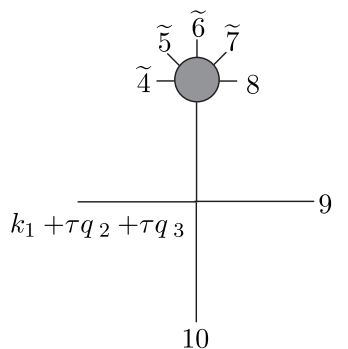

$(\mathrm{C} 2)$

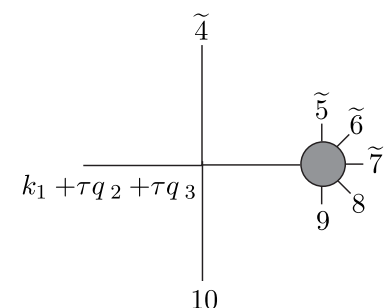

(C3)

Figure 6. All diagrams for semi-amplitude $A^{*}\left(k_{1}+\tau q_{2}+\tau q_{3}, \widetilde{4}, \widetilde{5}, \widetilde{6}, \widetilde{7}, 8,9,10\right)$ with hard pions belong to more than one sub-current.

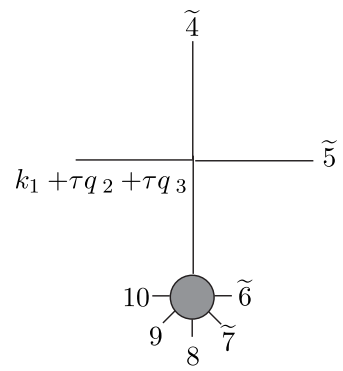

(A1)

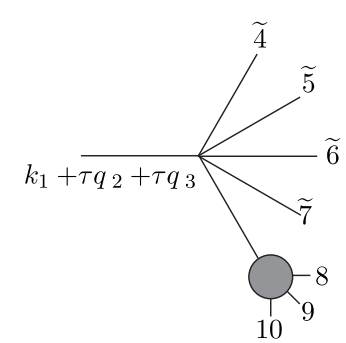

(A2)

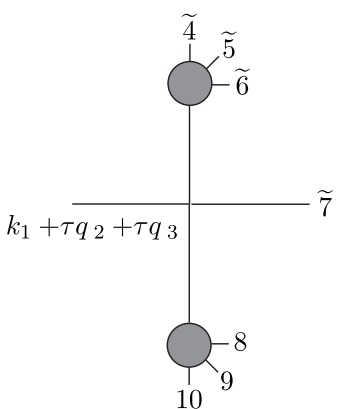

(A3)

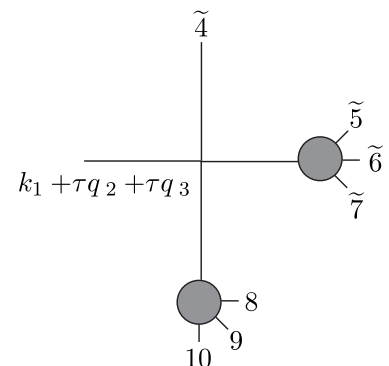

(A4)

Figure 7. All diagrams for semi-amplitude $A^{*}\left(k_{1}+\tau q_{2}+\tau q_{3}, \widetilde{4}, \widetilde{5}, \widetilde{6}, \widetilde{7}, 8,9,10\right)$ with all hard pions belong to a same sub-current.

leading soft behavior of other five diagrams apparently produces

$$
\begin{aligned}
A(1,8,9,10)[ & J^{(0)}(\widetilde{4}, \widetilde{5}, \widetilde{6}, \widetilde{7}, 8)+J(\widetilde{4}, \widetilde{5}, \widetilde{6}) J^{(0)}(\widetilde{7}) J^{(0)}(8)+J^{(0)}(\widetilde{4}) J^{(0)}(\widetilde{5}, \widetilde{6}, 7) J^{(0)}(8) \\
& \left.+J^{(0)}(\widetilde{4}) J^{(0)}(\widetilde{5}) J^{(0)}(\widetilde{6}, \widetilde{7}, 8)+J^{(0)}(\widetilde{4}) J^{(0)}(\widetilde{5}) J^{(0)}(\widetilde{6}) J^{(0)}(\widetilde{7}) J^{(0)}(8)\right]
\end{aligned}
$$

- Diagrams where all hard pions are included in a single sub-current.

This type is more subtle, consisting of diagrams given by figure 7 . In figure 7 . (A1), there is a semi-amplitude $A^{*}\left(k_{1}+\tau q_{2}+\tau q_{3}+\tau q_{4}+\tau q_{5}, \widetilde{6}, \widetilde{7}, 8,9,10\right)$. As iterative assumption, the behavior of lower-point is known, i.e., the leading behavior of this semi-amplitude satisfies 
eq. (4.9), as

$$
\begin{aligned}
& A^{*(0)}\left(k_{1}+\tau \sum_{i=2}^{5} q_{i}, \widetilde{6}, \widetilde{7}, 8,9,10\right) \\
& =\left.\left[A^{*}\left(k_{1}+\tau \sum_{i=2}^{5} q_{i}, \widetilde{6}, \widetilde{7},-\left(k_{1}+\tau \sum_{i=2}^{7} q_{i}\right)\right) \frac{-i}{\left(k_{1}+\tau \sum_{i=2}^{7} q_{i}\right)^{2}}\right]\right|_{\tau \rightarrow 0} J^{(0)}(8) A(1,8,9,10) \\
& +\left[J^{(0)}(\widetilde{6}, \widetilde{7}, 8)+J^{(0)}(\widetilde{6}) J^{(0)}(\widetilde{7}) J^{(0)}(8)\right] A(1,8,9,10),
\end{aligned}
$$

where the subcurrents $J(\widetilde{6}), J(\widetilde{7}), J(8)$ and the semi sub-current $J\left(k_{1}+\tau \sum_{i=2}^{5} q_{i}\right)$, with only one element, is 1 . Thus all their $\tau^{0}$ order should be 1 . Sum the first term in eq. (A.2) together with contributions from diagrams (A2), (A3), (A4), we arrive

$$
\begin{aligned}
& A(1,8,9,10) \\
& \times\left[i V_{4}\left(k_{1}+\tau q_{2}+\tau q_{3}, \widetilde{4}, \widetilde{5},-\left(k_{1}+\tau \sum_{i=2}^{5} q_{i}\right)\right) \frac{i}{\left(k_{1}+\tau \sum_{i=2}^{5} q_{i}\right)^{2}}\right. \\
& \quad \times A^{*}\left(k_{1}+\tau \sum_{i=2}^{5} q_{i}, \widetilde{6}, \widetilde{7},-\left(k_{1}+\tau \sum_{i=2}^{7} q_{i}\right)\right) \\
& +i V_{4}\left(k_{1}+\tau q_{2}+\tau q_{3}, \tau \sum_{i=4}^{6} q_{i}, \widetilde{7},-\left(k_{1}+\tau \sum_{i=2}^{7} q_{i}\right)\right) J(\widetilde{4}, \widetilde{5}, \widetilde{6}) \\
& +i V_{4}\left(k_{1}+\tau q_{2}+\tau q_{3}, \widetilde{4}, \tau \sum_{i=5}^{7} q_{i},-\left(k_{1}+\tau \sum_{i=2}^{7} q_{i}\right)\right) J(\widetilde{5}, \widetilde{6}, \widetilde{7}) \\
& \left.+i V_{6}\left(k_{1}+\tau q_{2}+\tau q_{3}, \widetilde{4}, \widetilde{5}, \widetilde{6}, \widetilde{7},-\left(k_{1}+\tau \sum_{i=2}^{7} q_{i}\right)\right)\right]\left.\frac{-i}{\left(k_{1}+\tau \sum_{i=2}^{7} q_{i}\right)^{2}}\right|_{\tau \rightarrow 0}
\end{aligned}
$$

The sum of the terms in the square brackets is a semi-amplitude $A^{*}\left(k_{1}+\tau q_{2}+\tau q_{3}, \widetilde{4}, \widetilde{5}, \widetilde{6}, \widetilde{7}\right.$, $\left.-\left(k_{1}+\tau \sum_{i=2}^{7} q_{i}\right)\right)$, thus the above expression is $A(1,8,9,10) J^{*(0)}\left(k_{1}+\tau q_{2}+\tau q_{3}, \widetilde{4}, \widetilde{5}, \widetilde{6}, \widetilde{7}\right)$. 
The last line in eq. (A.2) contributes a

$$
\begin{aligned}
& \left.A(1,8,9,10) i V_{4}\left(k_{1}+\tau q_{2}+\tau q_{3}, \widetilde{4}, \widetilde{5},-\left(k_{1}+\tau \sum_{i=2}^{5} q_{i}\right)\right) \frac{-i}{\left(k_{1}+\tau \sum_{i=2}^{5} q_{i}\right)^{2}}\right|_{\tau \rightarrow 0} \\
& \quad \times\left[J^{(0)}(\widetilde{6}, \widetilde{7}, 8)+J^{(0)}(\widetilde{6}) J^{(0)}(\widetilde{7}) J(8)\right] \\
& =A(1,8,9,10) J^{*(0)}\left(k_{1}+\tau q_{2}+\tau q_{3}, \widetilde{4}, \widetilde{5}\right)\left[J^{(0)}(\widetilde{6}, \widetilde{7}, 8)+J^{(0)}(\widetilde{6}) J^{(0)}(\widetilde{7}) J(8)\right],
\end{aligned}
$$

to the leading behavior of figure 7 . (A1). Thus all the diagrams in figure 7 are summed as

$$
\begin{gathered}
A(1,8,9,10)\left[J^{*(0)}\left(k_{1}+\tau q_{2}+\tau q_{3}, \widetilde{4}, \widetilde{5}, \widetilde{6}, \widetilde{7}\right) J(8)+J^{*(0)}\left(k_{1}+\tau q_{2}+\tau q_{3}, \widetilde{4}, \widetilde{5}\right) J^{(0)}(\widetilde{6}, \widetilde{7}, 8)\right. \\
\left.\quad+J^{*(0)}\left(k_{1}+\tau q_{2}+\tau q_{3}, \widetilde{4}, \widetilde{5}\right) J^{(0)}(\widetilde{6}) J^{(0)}(\widetilde{7}) J(8)\right]
\end{gathered}
$$

Putting coupling constants back, eq. (A.1) and eq. (A.5), corresponding to figure 6 and figure 7 respectively, result in the expected soft behavior of the semi-amplitude $A^{*}\left(k_{1}+\tau q_{2}+\tau q_{3}, \widetilde{4}, \widetilde{5}, \widetilde{6}, \widetilde{7}, 8,9,10\right)$ shown in eq. (4.9).

\section{A.2 General proof}

From the example, we find that diagrams of the Berends-Giele recursion expansion of a semi-amplitude are classified into two categories. This pattern can be easily generalized to an arbitrary point semi-amplitude $A^{*}\left(\left(k_{1}+\sum_{i=2}^{2 m-1} q_{i}\right), \widetilde{2 m}, \cdots, \widetilde{2 k+1}, 2 k+2 \cdots, 2 n\right)$ : i) diagrams with hard pions living in more than one sub-current, ii) diagrams with all hard pions in a single sub-current. Now let us discuss these two cases separately. ${ }^{6}$

i) Diagrams where on-shell hard pions $2 k+2, \ldots, 2 n$ belong to different sub-currents, figure $5(\mathrm{~A})$ :

The leading soft behavior of such a diagram is

$$
\left.i\left(\tau \sum_{l=0}^{I} Q_{S_{2 l+1}}+\sum_{m=0}^{J} P_{H_{2 m+1}}\right)^{2}\right|_{\tau=0}\left[\prod_{i=1}^{2 I} J^{(0)}\left(S_{i}\right)\right] J^{(0)}\left(S_{2 I+1}, H_{1}\right)\left[\prod_{j=2}^{2 J+1} J\left(H_{j}\right)\right],
$$

where $\tau Q_{S_{2 l+1}}$ means summing over momenta of all soft pions in the set $S_{2 l+1}$, and the current $J\left(H_{i}\right)$ is of order $\tau^{0}$ itself. Since the sub-current $J\left(S_{2 I+1}, H_{1}\right)$ does not contain all hard pions, it satisfies the soft behavior eq. (4.1). The $\tau^{0}$ behavior of figure $5(\mathrm{~A})$ is

$$
J^{(0)}\left(S_{2 I+1}, 2 k+2\right) \prod_{i=1}^{2 I} J^{(0)}\left(S_{i}\right)\left[i\left(\sum_{m=1}^{J} P_{H_{2 m+1}}\right)^{2} \prod_{j=1}^{2 J+1} J\left(H_{j}\right)\right] .
$$

\footnotetext{
${ }^{6}$ Again, we omit coupling constants and put them back in the final expression.
} 
Evidently, leading soft behavior of this type is written as

$$
\sum_{\text {Divisions }^{(A)}(S)} J^{(0)}\left(S_{2 I+1}, 2 k+2\right) \prod_{i=1}^{2 I} J^{(0)}\left(S_{i}\right)\left[i \sum_{J=1}^{n-1-k} \sum_{\text {Divisions }(\{H\})}\left(\sum_{m=1}^{J} P_{H_{2 m+1}}\right)^{2} \prod_{j=1}^{2 J+1} J\left(H_{j}\right)\right]
$$

Here "Divisions ${ }^{(A)}(S)$ " stands for all possible divisions of $\widetilde{2 m}, \ldots, \widetilde{2 k+1}$. Among those divisions, the last subset contains soft pions of even number while all other subsets contain soft pions of odd number. The summation in square brackets is Berends-Giele recursion expansions (i.e., the sum of all Feynman diagrams) of the amplitude $A(1,2 k+2, \cdots, 2 n)$, thus the above equation finally provides

$$
\mathbb{S}^{*(0) A}\left(k_{1}+\tau \sum_{i=2}^{2 m-1} q_{i}, 2 k+2\right) A(1,2 k+2, \cdots, 2 n),
$$

in which

$$
\mathbb{S}^{*(0) A}\left(k_{1}+\tau \sum_{i=2}^{2 m-1} q_{i}, 2 k+2\right) \equiv \sum_{\mathcal{D}^{A}} \prod_{i=1}^{\mathcal{N}\left(\mathcal{D}^{A}\right)} J^{(0)}\left(\left\{\mathcal{S}_{\mathcal{D}^{A}}^{i}\right\}\right)
$$

where we have summed over all divisions $\mathcal{D}^{A}$ of $\{\widetilde{2 m}, \widetilde{2 m+1}, \cdots, \widetilde{2 k+1}, 2 k+2\}$ such that each subset contains elements of odd number.

ii) Diagrams where on-shell hard pions $2 k+2, \ldots, 2 n$ belong to a single sub-current, figure $5(\mathrm{~B})$ :

Such diagram contributes a term

$$
\left.\left[i\left(\tau \sum_{l=0}^{I} Q_{S_{2 l+1}}+P_{H}\right)^{2} \prod_{i=1}^{2 I} J\left(S_{j}\right) \frac{i}{\left(k_{1}+\tau \sum_{i=1}^{2 m-1} q_{i}\right)^{2}} A^{*}\left(\left(k_{1}+\tau \sum_{i=1}^{2 m-1} q_{i}\right), S_{2 I+1}, H\right)\right]\right|_{\tau=0} .
$$

Considering the soft behavior of $A^{*}\left(\left(k_{1}+\tau \sum_{i=1}^{2 m-1} q_{i}\right), S_{2 I+1}, H\right)$, from inductive assumption for lower point semi-amplitudes in eq. (4.9), we have

$$
A^{*(0)}\left(\left(k_{1}+\tau \sum_{i=1}^{2 m-1} q_{i}\right), S_{2 I+1}, H\right)=\sum_{\mathcal{D}^{\prime}}\left(J^{*(0)}\left(S_{\mathcal{D}^{\prime}}^{1}\right) \prod_{l=2}^{\mathcal{N}\left(\mathcal{D}^{\prime}\right)} J^{(0)}\left(S_{\mathcal{D}^{\prime}}^{l}\right)\right) A(1,2 k+2, \cdots, 2 n),
$$

where $\mathcal{D}^{\prime}$ are divisions of the ordered set $\left\{\left(k_{1}+\tau \sum_{i=1}^{2 m-1} q_{i}\right), S_{2 I+1}, 2 k+2\right\}$. The sum of 
the leading behaviors of diagrams of figure 5 (B) type contributes

$$
\begin{aligned}
& \left\{A(1,2 k+2, \cdots, 2 n) \sum_{I} \sum_{\mathcal{D}} i\left(\tau \sum_{l=0}^{I} P_{S_{2 l+1}}+P_{H}\right)^{2}\left[\prod_{j=1}^{2 I} J\left(S_{j}\right)\right]\right. \\
& \times\left[\sum_{\mathcal{D}^{\prime}} \frac{i}{\left(k_{1}+\tau \sum_{i=1}^{2 m-1} q_{i}\right)^{2}} A^{*}\left(\left(k_{1}+\tau \sum_{i=1}^{2 m-1} q_{i}\right), S_{\mathcal{D}^{\prime}}^{1},-\left(k_{1}+\tau \sum_{i=1}^{2 m-1} q_{i}+P_{S_{\mathcal{D}^{\prime}}^{1}}\right)\right)\right. \\
& \left.\left.\times \frac{i}{\left(k_{1}+\tau \sum_{i=1}^{2 m-1} q_{i}+P_{S_{\mathcal{D}^{\prime}}}\right)^{2}} \prod_{l=2}^{\mathcal{N}\left(\mathcal{D}^{\prime}\right)} J\left(S_{\mathcal{D}^{\prime}}^{l}\right)\right]\right\}\left.\right|_{\tau \rightarrow 0},
\end{aligned}
$$

where $\mathcal{D}$ denote all possible divisions of soft pions $\{\widetilde{2 m}, \widetilde{2 m+1}, \cdots, \widetilde{2 k+1}\} \rightarrow\left\{S_{1}\right\} \cdots$ $\left\{S_{2 I}\right\},\left\{S_{2 I+1}\right\}$. The sum over divisions $\mathcal{D}$ and $\mathcal{D}^{\prime}$ can be rearranged by collecting those terms which have a same last soft pion in the first subset $\left\{S_{\mathcal{D}^{\prime}}^{1}\right\}$ of $\mathcal{D}^{\prime}$ (in the example of the previous subsection, eq. (A.3) is a collection of terms with the pion 7 as the last element of $\left\{S_{\mathcal{D}^{\prime}}^{1}\right\}$, while eq. (A.4) is a collection of terms with 5 as the last element of $\left\{S_{\mathcal{D}^{\prime}}^{1}\right\}$ ). After this rearrangement, eq. (A.13) turns out

$$
\begin{aligned}
& \left\{( 1 , 2 k + 2 , \cdots , 2 n ) \sum _ { a = m } ^ { k } \left[\sum_{I=4}^{2 a-2 m+4} \sum_{\mathcal{D}_{2 a+1}} i\left(\tau \sum_{l=0}^{I-1} P_{S_{2 l+1}}+P_{H}\right)^{2}\left[\prod_{j=1}^{I-2} J\left(S_{\mathcal{D}_{2 a+1}}^{j}\right)\right]\right.\right. \\
& \left.\times \frac{i}{\left(k_{1}+\tau \sum_{i=1}^{2 m-1} q_{i}\right)^{2}} A^{*}\left(\left(k_{1}+\tau \sum_{i=1}^{2 m-1} q_{i}\right), S_{\mathcal{D}_{2 a+1}}^{I-1},-\left(k_{1}+\tau \sum_{i=1}^{2 a+1} q_{i}\right)\right)\right] \frac{i}{\left(k_{1}+\tau \sum_{i=1}^{2 a+1} q_{i}\right)^{2}} \\
& \left.\times \sum_{\mathcal{D}^{\prime} \in \text { Divisions }\{2 a+2, \cdots, 2 m+1\}} \prod_{l=1}^{\mathcal{N}\left(\mathcal{D}^{\prime}\right)} J\left(S_{\mathcal{D}^{\prime}}^{l}\right)\right\}\left.\right|_{\tau \rightarrow 0} .
\end{aligned}
$$

Note that the second line in the brackets is the sub-current $J^{*}\left(S_{\mathcal{D}_{2 a+1}}^{I-1},-\left(k_{1}+\tau \sum_{i=1}^{2 a+1} q_{i}\right)\right)$ where $-\left(k_{1}+\tau \sum_{i=1}^{2 a+1} q_{i}\right)$ is also off-shell. All the factors given in the square brackets thus produce a semi-amplitude $A^{*}\left(k_{1}+\tau \widetilde{2 m-1} \sum_{i=2}^{2 m} q_{i} \widetilde{2 m}, \cdots, \widetilde{2 a+1},-\left(k_{1}+\tau \sum_{i=1}^{2 a+1} q_{i}\right)\right)$ which becomes a semi-current $J^{*}\left(k_{1}+\tau \sum_{i=2}^{2 m-1} q_{i}, \widetilde{2 m}, \cdots, \widetilde{2 a+1}\right)$ if multiplied by a factor 


$$
\begin{aligned}
& \frac{i}{\left(k_{1}+\tau \sum_{i=1}^{2 a+1} q_{i}\right)^{2}} \text {. The expression eq. (A.13) then becomes } \\
& A(1,2 k+2, \cdots, 2 n) \\
& \quad \times\left[\sum_{a=m}^{k} J^{*(0)}\left(k_{1}+\tau \sum_{i=2}^{2 m-1} q_{i}, \widetilde{2 m}, \cdots, \widetilde{2 a+1}\right)_{\mathcal{D}^{\prime} \in \text { Divisions }\{2 a+2, \cdots, 2 m+1\}} \prod_{l=1}^{\mathcal{N}\left(\mathcal{D}^{\prime}\right)} J\left(S_{\mathcal{D}^{\prime}}^{l}\right)\right] .
\end{aligned}
$$

Terms in the square brackets can be expressed as

$$
\mathbb{S}^{*(0) B}\left(k_{1}+\tau \sum_{i=2}^{2 m-1} q_{i}, 2 k+2\right) \equiv \sum_{\mathcal{D}^{B}} \prod_{l=1}^{\mathcal{N}\left(\mathcal{D}^{B}\right)} J^{(0)}\left(\left\{\mathcal{S}_{\mathcal{D}^{B}}^{l}\right\}\right)
$$

where $\mathcal{D}^{B}$ stands for all possible divisions of $\left\{k_{1}+\tau \sum_{i=2}^{2 m-1} q_{i}, \widetilde{2 m}, \cdots, \widetilde{2 k+1}, 2 k+2\right\}$ such that each subset contains element of odd number and the first subset contains more than one element. Therefore eq. (A.13) can be written as

$$
\mathbb{S}^{*(0) B}\left(k_{1}+\tau \sum_{i=2}^{2 m-1} q_{i}, 2 k+2\right) A(1,2 k+2, \cdots, 2 n) .
$$

Finally, summing both types of diagrams together and plugging coupling constants back, we obtain the leading behavior

$$
\begin{aligned}
& A^{*}\left(\left(k_{1}+\sum_{i=2}^{2 m-1} q_{i}\right), \widetilde{2 m}, \cdots, \widetilde{2 k+1}, 2 k+2 \cdots, 2 n\right) \\
& =\left[\mathbb{S}^{*(0) A}\left(k_{1}+\tau \sum_{i=2}^{2 m-1} q_{i}, 2 k+2\right)+\mathbb{S}^{*(0) B}\left(k_{1}+\tau \sum_{i=2}^{2 m-1} q_{i}, 2 k+2\right)\right] A(1,2 k+2, \cdots, 2 n) \\
& =\mathbb{S}^{*(0)}\left(k_{1}+\tau \sum_{i=2}^{2 m-1} q_{i}, 2 k+2\right) A(1,2 k+2, \cdots, 2 n)
\end{aligned}
$$

which is the expected behavior eq. (4.7). 


\section{B Sextuple behavior}

The sextuple soft factor for the color-like ordered NLSM amplitudes $A(1,2, \widetilde{3}, \widetilde{4}, \ldots, \widetilde{8}, 9$, $\ldots, 2 n)$ is

$$
\begin{aligned}
& \mathbb{S}_{2,8}^{(0)}=+\frac{1}{8 F^{6}} \frac{1}{4}\left[1-\frac{s_{24}}{s_{2 \mid 3,4}}-\frac{s_{97}}{s_{9 \mid 7,8}}+\frac{s_{28}}{s_{2 \mid 3,8}}+\frac{s_{93}}{s_{9 \mid 3,8}}\right] \\
& +\frac{1}{8 F^{6}}\left\{\left(\frac{s_{24}}{s_{2 \mid 3,4}}-\frac{1}{2}\right)\left(\frac{s_{26}}{s_{2 \mid 3,6}}-\frac{1}{2}\right)\left(\frac{s_{28}}{s_{2 \mid 3,8}}-\frac{1}{2}\right)+\left(\frac{s_{97}}{s_{9 \mid 7,8}}-\frac{1}{2}\right)\left(\frac{s_{95}}{s_{9 \mid 5,8}}-\frac{1}{2}\right)\left(\frac{s_{93}}{s_{9 \mid 3,8}}-\frac{1}{2}\right)\right. \\
& +\left(\frac{s_{24}}{s_{2 \mid 3,4}}-\frac{1}{2}\right)\left(\frac{s_{26}}{s_{2 \mid 3,6}}-\frac{1}{2}\right)\left(\frac{s_{79}}{s_{9 \mid 7,8}}-\frac{1}{2}\right)+\left(\frac{s_{24}}{s_{2 \mid 3,4}}-\frac{1}{2}\right)\left(\frac{s_{59}}{s_{9 \mid 5,8}}-\frac{1}{2}\right)\left(\frac{s_{79}}{s_{9 \mid 7,8}}-\frac{1}{2}\right) \\
& -\frac{1}{2}\left(\frac{s_{24}}{s_{2 \mid 3,4}}-\frac{1}{2}\right)\left(\frac{s_{28}}{s_{2 \mid 3,8}}-\frac{1}{2}\right)-\frac{1}{2}\left(\frac{s_{26}}{s_{2 \mid 3,6}}-\frac{1}{2}\right)\left(\frac{s_{28}}{s_{2 \mid 3,8}}-\frac{1}{2}\right) \\
& -\frac{1}{2}\left(\frac{s_{79}}{s_{9 \mid 7,8}}-\frac{1}{2}\right)\left(\frac{s_{93}}{s_{9 \mid 3,8}}-\frac{1}{2}\right)-\frac{1}{2}\left(\frac{s_{59}}{s_{9 \mid 5,8}}-\frac{1}{2}\right)\left(\frac{s_{93}}{s_{9 \mid 3,8}}-\frac{1}{2}\right) \\
& \left.-\frac{1}{2}\left(\frac{s_{26}}{s_{2 \mid 3,6}}-\frac{1}{2}\right)\left(\frac{s_{79}}{s_{9 \mid 7,8}}-\frac{1}{2}\right)-\frac{1}{2}\left(\frac{s_{24}}{s_{2 \mid 3,4}}-\frac{1}{2}\right)\left(\frac{s_{59}}{s_{9 \mid 5,8}}-\frac{1}{2}\right)\right\} \\
& +\frac{s_{35}}{8 F^{6} s_{3 ; 5}}\left\{\left(\frac{s_{26}}{s_{2 \mid 3,6}}-\frac{1}{2}\right)\left(\frac{s_{28}}{s_{2 \mid 3,8}}-\frac{1}{2}\right)+\left(\frac{s_{79}}{s_{9 \mid 7,8}}-\frac{1}{2}\right)\left(\frac{s_{9 \mid 3,5}}{s_{9 \mid 3,8}}-\frac{1}{2}\right)\right. \\
& \left.+\left(\frac{s_{26}}{s_{2 \mid 3,6}}-\frac{1}{2}\right)\left(\frac{s_{79}}{s_{9 \mid 7,8}}-\frac{1}{2}\right)+\left(\frac{s_{28}}{s_{2 \mid 3,8}}-\frac{1}{2}\right)\left(\frac{s_{9 \mid 3,5}}{s_{9 \mid 3,8}}-\frac{1}{2}\right)-\frac{s_{28}}{s_{2 \mid 3,8}} \frac{s_{9 \mid 3,5}}{s_{9 \mid 3,8}}\right\} \\
& +\frac{s_{46}}{8 F^{6} s_{4 ; 6}}\left\{\left(\frac{s_{2 \mid 4,6}}{s_{2 \mid 3,6}}-\frac{1}{2}\right)\left(\frac{s_{28}}{s_{2 \mid 3,8}}-\frac{1}{2}\right)+\left(\frac{s_{79}}{s_{9 \mid 7,8}}-\frac{1}{2}\right)\left(\frac{s_{39}}{s_{9 \mid 3,8}}-\frac{1}{2}\right)\right. \\
& \left.+\left(\frac{s_{2 \mid 4,6}}{s_{2 \mid 3,6}}-\frac{1}{2}\right)\left(\frac{s_{79}}{s_{9 \mid 7,8}}-\frac{1}{2}\right)+\left(\frac{s_{28}}{s_{2 \mid 3,8}}-\frac{1}{2}\right)\left(\frac{s_{39}}{s_{9 \mid 3,8}}-\frac{1}{2}\right)-\frac{s_{28}}{s_{2 \mid 3,8}} \frac{s_{39}}{s_{9 \mid 3,8}}\right\} \\
& +\frac{s_{57}}{8 F^{6} s_{5 ; 7}}\left\{\left(\frac{s_{24}}{s_{2 \mid 3,4}}-\frac{1}{2}\right)\left(\frac{s_{28}}{s_{2 \mid 3,8}}-\frac{1}{2}\right)+\left(\frac{s_{9 \mid 5,7}}{s_{9 \mid 5,8}}-\frac{1}{2}\right)\left(\frac{s_{39}}{s_{9 \mid 3,8}}-\frac{1}{2}\right)\right. \\
& \left.+\left(\frac{s_{24}}{s_{2 \mid 3,4}}-\frac{1}{2}\right)\left(\frac{s_{9 \mid 5,7}}{s_{9 \mid 5,8}}-\frac{1}{2}\right)+\left(\frac{s_{28}}{s_{2 \mid 3,8}}-\frac{1}{2}\right)\left(\frac{s_{39}}{s_{9 \mid 3,8}}-\frac{1}{2}\right)-\frac{s_{28}}{s_{2 \mid 3,8}} \frac{s_{39}}{s_{9 \mid 3,8}}\right\} \\
& +\frac{s_{68}}{8 F^{6} s_{6 ; 8}}\left\{\left(\frac{s_{24}}{s_{2 \mid 3,4}}-\frac{1}{2}\right)\left(\frac{s_{2 \mid 6,8}}{s_{2 \mid 3,8}}-\frac{1}{2}\right)+\left(\frac{s_{59}}{s_{9 \mid 5,8}}-\frac{1}{2}\right)\left(\frac{s_{39}}{s_{9 \mid 3,8}}-\frac{1}{2}\right)\right. \\
& \left.+\left(\frac{s_{24}}{s_{2 \mid 3,4}}-\frac{1}{2}\right)\left(\frac{s_{59}}{s_{9 \mid 5,8}}-\frac{1}{2}\right)+\left(\frac{s_{2 \mid 6,8}}{s_{2 \mid 3,8}}-\frac{1}{2}\right)\left(\frac{s_{39}}{s_{9 \mid 3,8}}-\frac{1}{2}\right)-\frac{s_{2 \mid 6,8}}{s_{2 \mid 3,8}} \frac{s_{39}}{s_{9 \mid 3,8}}\right\} \\
& -\frac{s_{37}}{8 F^{6} s_{3 ; 7}}\left(\frac{s_{28}}{s_{2 \mid 3,8}}+\frac{s_{9 \mid 3,7}}{s_{9 \mid 3,8}}-1\right)-\frac{s_{48}}{8 F^{6} s_{4 ; 8}}\left(\frac{s_{2 \mid 4,8}}{s_{2 \mid 3,8}}+\frac{s_{39}}{s_{9 \mid 3,8}}-1\right) \\
& +\frac{s_{35} s_{68}}{8 F^{6} s_{3 ; 5} s_{6 ; 8}}\left(\frac{s_{2 \mid 6,8}}{s_{2 \mid 3,8}}+\frac{s_{9 \mid 3,5}}{s_{9 \mid 3,8}}-1\right) \\
& +\frac{s_{35} s_{7 \mid 3,5}}{8 F^{6} s_{3 ; 5} s_{3 ; 7}}\left(\frac{s_{28}}{s_{2 \mid 3,8}}-\frac{s_{89}}{s_{9 \mid 3,8}}\right)+\frac{s_{46} s_{37}}{8 F^{6} s_{4 ; 6} s_{3 ; 7}}\left(\frac{s_{28}}{s_{2 \mid 3,8}}-\frac{s_{89}}{s_{9 \mid 3,8}}\right)+\frac{s_{57} s_{3 \mid 5,7}}{8 F^{6} s_{5 ; 7} s_{3 ; 7}}\left(\frac{s_{28}}{s_{2 \mid 3,8}}-\frac{s_{89}}{s_{9 \mid 3,8}}\right) \\
& +\frac{s_{46} s_{8 \mid 4,6}}{8 F^{6} s_{4 ; 6} s_{4 ; 8}}\left(\frac{s_{39}}{s_{9 \mid 3,8}}-\frac{s_{23}}{s_{2 \mid 3,8}}\right)+\frac{s_{57} s_{48}}{8 F^{6} s_{5 ; 7} s_{4 ; 8}}\left(\frac{s_{39}}{s_{9 \mid 3,8}}-\frac{s_{23}}{s_{2 \mid 3,8}}\right)+\frac{s_{68} s_{4 \mid 6,8}}{8 F^{6} s_{6 ; 8} s_{4 ; 8}}\left(\frac{s_{39}}{s_{9 \mid 3,8}}-\frac{s_{23}}{s_{2 \mid 3,8}}\right) \text {, }
\end{aligned}
$$

where $s_{i \mid j, k}=2 p_{i} \cdot\left(p_{j}+\cdots+p_{k}\right)$ and $s_{i ; j}=\left(p_{i}+\cdots+p_{j}\right)^{2}$. 
Open Access. This article is distributed under the terms of the Creative Commons Attribution License (CC-BY 4.0), which permits any use, distribution and reproduction in any medium, provided the original author(s) and source are credited.

\section{References}

[1] S.R. Coleman, J. Wess and B. Zumino, Structure of phenomenological Lagrangians. 1, Phys. Rev. 177 (1969) 2239 [inSPIRE].

[2] C.G. Callan Jr., S.R. Coleman, J. Wess and B. Zumino, Structure of phenomenological Lagrangians. 2, Phys. Rev. 177 (1969) 2247 [INSPIRE].

[3] N. Arkani-Hamed, F. Cachazo and J. Kaplan, What is the Simplest Quantum Field Theory?, JHEP 09 (2010) 016 [arXiv:0808.1446] [INSPIRE].

[4] S.L. Adler, Consistency conditions on the strong interactions implied by a partially conserved axial vector current, Phys. Rev. 137 (1965) B1022 [INSPIRE].

[5] L. Susskind and G. Frye, Algebraic aspects of pionic duality diagrams, Phys. Rev. D 1 (1970) 1682 [INSPIRE].

[6] F. Cachazo, S. He and E.Y. Yuan, New Double Soft Emission Theorems, Phys. Rev. D 92 (2015) 065030 [arXiv:1503.04816] [INSPIRE].

[7] Y.-J. Du and H. Lüo, On single and double soft behaviors in NLSM, JHEP 08 (2015) 058 [arXiv: 1505.04411] [INSPIRE].

[8] I. Low, Double Soft Theorems and Shift Symmetry in Nonlinear $\sigma$-models, Phys. Rev. D 93 (2016) 045032 [arXiv: 1512.01232] [INSPIRE].

[9] S. He, Z. Liu and J.-B. Wu, Scattering Equations, Twistor-string Formulas and Double-soft Limits in Four Dimensions, JHEP 07 (2016) 060 [arXiv: 1604.02834] [INSPIRE].

[10] R.H. Boels and W. Wormsbecher, Spontaneously broken conformal invariance in observables, arXiv: 1507.08162 [INSPIRE].

[11] Y.-t. Huang and C. Wen, Soft theorems from anomalous symmetries, JHEP 12 (2015) 143 [arXiv: 1509.07840] [INSPIRE].

[12] P. Di Vecchia, R. Marotta, M. Mojaza and J. Nohle, New soft theorems for the gravity dilaton and the Nambu-Goldstone dilaton at subsubleading order, Phys. Rev. D 93 (2016) 085015 [arXiv: 1512.03316] [INSPIRE].

[13] W.-M. Chen, Y.-t. Huang and C. Wen, New Fermionic Soft Theorems for Supergravity Amplitudes, Phys. Rev. Lett. 115 (2015) 021603 [arXiv:1412.1809] [INSPIRE].

[14] R. Kallosh, Nonlinear (Super)Symmetries and Amplitudes, arXiv:1609.09123 [INSPIRE].

[15] R. Kallosh, A. Karlsson and D. Murli, Origin of Soft Limits from Nonlinear Supersymmetry in Volkov-Akulov Theory, arXiv:1609.09127 [INSPIRE].

[16] T. McLoughlin and D. Nandan, Multi-Soft gluon limits and extended current algebras at null-infinity, arXiv:1610.03841 [INSPIRE].

[17] A.E. Lipstein, Soft Theorems from Conformal Field Theory, JHEP 06 (2015) 166 [arXiv: 1504.01364] [INSPIRE].

[18] R. Britto, F. Cachazo and B. Feng, New recursion relations for tree amplitudes of gluons, Nucl. Phys. B 715 (2005) 499 [hep-th/0412308] [INSPIRE]. 
[19] R. Britto, F. Cachazo, B. Feng and E. Witten, Direct proof of tree-level recursion relation in Yang-Mills theory, Phys. Rev. Lett. 94 (2005) 181602 [hep-th/0501052] [INSPIRE].

[20] R. Kleiss and H. Kuijf, Multi-Gluon Cross-sections and Five Jet Production at Hadron Colliders, Nucl. Phys. B 312 (1989) 616 [inSPIRE].

[21] K. Kampf, J. Novotny and J. Trnka, Recursion relations for tree-level amplitudes in the $\mathrm{SU}(N)$ nonlinear $\sigma$-model, Phys. Rev. D 87 (2013) 081701 [arXiv:1212.5224] [INSPIRE].

[22] K. Kampf, J. Novotny and J. Trnka, Tree-level Amplitudes in the Nonlinear $\sigma$-model, JHEP 05 (2013) 032 [arXiv: 1304.3048] [INSPIRE].

[23] C. Cheung, K. Kampf, J. Novotny, C.-H. Shen and J. Trnka, On-Shell Recursion Relations for Effective Field Theories, Phys. Rev. Lett. 116 (2016) 041601 [arXiv:1509.03309] [INSPIRE].

[24] H. Lüo and C. Wen, Recursion relations from soft theorems, JHEP 03 (2016) 088 [arXiv: 1512.06801] [INSPIRE].

[25] F. Cachazo, S. He and E.Y. Yuan, Scattering equations and Kawai-Lewellen-Tye orthogonality, Phys. Rev. D 90 (2014) 065001 [arXiv:1306.6575] [INSPIRE].

[26] F. Cachazo, S. He and E.Y. Yuan, Scattering of Massless Particles in Arbitrary Dimensions, Phys. Rev. Lett. 113 (2014) 171601 [arXiv:1307.2199] [INSPIRE].

[27] F. Cachazo, S. He and E.Y. Yuan, Scattering of Massless Particles: Scalars, Gluons and Gravitons, JHEP 07 (2014) 033 [arXiv:1309.0885] [INSPIRE].

[28] F. Cachazo, S. He and E.Y. Yuan, Scattering Equations and Matrices: From Einstein To Yang-Mills, DBI and NLSM, JHEP 07 (2015) 149 [arXiv:1412.3479] [INSPIRE].

[29] F. Cachazo, P. Cha and S. Mizera, Extensions of Theories from Soft Limits, JHEP 06 (2016) 170 [arXiv: 1604.03893] [INSPIRE].

[30] J.J.M. Carrasco, C.R. Mafra and O. Schlotterer, Abelian Z-theory: NLSM amplitudes and $\alpha^{\prime}$-corrections from the open string, arXiv:1608.02569 [INSPIRE]. 